\title{
Quantifying Three-Dimensional Residual Stress Distributions Using Spatially-Resolved Diffraction Measurements and Finite Element Based Data Reduction
}

\author{
Jun-Sang Park ${ }^{2}{ }^{*}$ \\ Ulrich Lienert ${ }^{3}$ \\ Paul R. Dawson ${ }^{1}$ \\ Matthew P. Miller ${ }^{1 \dagger}$ \\ ${ }^{1}$ Sibley School of Mechanical and Aerospace Engineering, Cornell University, Ithaca NY \\ ${ }^{2}$ Advanced Photon Source, Argonne National Laboratory, Argonne, IL \\ ${ }^{3}$ Deutsches Elektronen-Synchrotron, Hamburg, Germany \\ Published in Experimental Mechanics, vol 53, number 9, p.1491-1507, 2013 (DOI: \\ http://dx.doi.org/10.1007/s11340-013-9771-0).
}

\begin{abstract}
Residual stress can play a significant role in the processing and performance of an engineered metallic component. The stress state within a polycrystalline part can vary significantly between its surface and its interior. To measure three-dimensional (3D) residual stress fields, a synchrotron x-ray diffraction-based experimental technique capable of non-destructively measuring a set of lattice strain pole figures (SPFs) at various surface and internal points within a component was developed. The resulting SPFs were used as input for a recently developed bi-scale optimization scheme [31] that combines crystal-scale measurements and continuum-scale constraints to determine the 3D residual stress field in the component. To demonstrate this methodology, the 3D residual stress distribution was evaluated for an interference-fit sample fabricated from a low solvus high refractory (LSHR) polycrystalline Ni-base superalloy. keywords: residual stress, high energy synchrotron x-ray, diffraction, lattice strain, multi-scale, conical slits
\end{abstract}

\section{Introduction}

It is well-known that various processing methods used on polycrystalline metallic materials give rise to three-dimensional (3D) residual stress fields that can vary significantly from the surface of a component to its core. Machining [20], forming [18], and shot peening [22] [8] are well-known examples where desired or undesired residual stress fields are introduced. Thermomechanical processes such as heat treatment, interference fitting [43], forging [45], and welding [1] [38], or themochemical processes such as nitriding and carburizing [4] also introduce residual stresses that change appreciably below the surface of a component. Understanding the spatial variation of stress is crucial for planning subsequent manufacturing processes or for predicting the life of the component. Neutron and high energy x-ray diffraction are especially effective non-destructive methods for penetrating metallic components to make subsurface stress measurements. With their explicit connection to microscale lattice distortion, diffraction data also hold potential for substantiating the macroscopic stress at a material or continuum point with the underlying crystal-scale stress states. High energy synchrotron x-rays and high speed area detectors have revolutionized the ability for making many strain measurements in many directions at multiple points in a workpiece in a very short amount of time. The challenge

\footnotetext{
*Electronic address: parkjseaps.anl.gov

${ }^{\dagger}$ Electronic address: mpm 4 @ cornell.edu
} 
becomes merging these data into a comprehensive description of the residual stress field within the component. Our group has developed a finite element-based method for accomplishing this integration [31]. Thousands of lattice strain measurements from throughout a sample containing a distribution of stress are made using high energy synchrotron $\mathrm{x}$-ray diffraction. At each diffraction volume, lattice strain pole figures are constructed from the measured strain data. The lattice strain pole figures for a diffraction volume contain sufficient data to determine a strain orientation distribution function for that volume. Here, we define that distribution over the fundamental region of Rodrigues space using finite element (FE) interpolation[16] [25]. From the strain distribution, a crystal stress distribution is readily computed using Hooke's law. The crystal stress distribution, in turn, is approximated with a harmonic expansion, which provides a very compact representation of the distribution. The crystal stress distribution can be integrated over the fundamental region to provide an average value of stress for the entire diffraction volume. These averages or diffraction-volume crystal stresses are used as input data to define a continuum stress distribution. The continuum stress distribution is represented with FE interpolation functions over the component and are required to be smooth. Further, this distribution is required to obey equilibrium locally and satisfy the boundary conditions over the surface of the component. Altogether, the unknown fitting parameters in the method are the harmonic coefficients for the crystal stress distribution at each diffraction volume and the nodal point values for the FE interpolation of the continuum stress distribution. The complete set of fitting parameters are determined using an optimization method that minimizes the difference between the continuum stress distribution and an interpolation of the diffraction-volume crystal stresses using the strain pole figure data as input.

In this paper, we describe the application of our framework to a polycrystalline nickel component containing a fully three-dimensional distribution of stress. We pay particular attention to describing details of the experimental method of using a set of "conical" $\mathrm{x}$-ray slits to segregate the diffracted x-ray beam coming from each subsurface diffraction volume. The structure of this paper is as follows. An overview of diffraction-based methods and the specifics of our diffraction measurements for measuring lattice strain pole figures (SPFs) are presented in $\$ 2$. The conical slits that enable the measurement of SPFs at the internal points within a sample are described in 8 and the experimental method used to measure the lattice strains at the internal points within the sample is described in \&4 Our data reduction method for the lattice strains is presented in $₫ 5$ and the application of the bi-scale FE framework, originally described in detail in McNelis et al. [31] and in Demir et al. [12], is outlined in $\$ 6$. Finally, the strain pole figure data and the 3D residual stress field data are presented and discussed in $\$ 7$ with conclusions and an outlook presented in $\$ 8$

\section{Diffraction Methods for Determining Residual Stress}

The governing equation for diffraction in crystalline materials is Bragg's law, which relates the wavelength of the probing radiation, $\lambda$, to the direction of the diffracted beam and the spacing of the diffracting lattice planes,

$$
\lambda=2 d_{\mathbf{n}} \sin \theta_{\mathbf{n}}
$$

Here, $\lambda$ is the wavelength of the incident beam, $d_{\mathbf{n}}$ is the lattice spacing for a family of crystallographic planes where $\mathbf{n}$ is the plane normal of the family of planes, and $\theta_{\mathbf{n}}$ is the corresponding Bragg angle. A particular family of crystallographic planes is denoted as $\{h k l\}$ from here on. For a given orientation of a polycrystalline aggregate with respect to the incident beam, all families of crystallographic planes that satisfy the Equation 3 (Bragg's law) will diffract and produce diffraction peaks. The value of $d_{\mathbf{n}}$ depends on the crystal symmetry, the lattice parameters of the crystal, and the $\{h k l\}$. For a monochromatic beam, changes in lattice spacing can be determined by monitoring $\theta_{\mathbf{n}}$. Diffraction is one of the earliest residual stress estimation methods and is used extensively. Bragg's law governs diffraction of neutrons [2], as well as laboratory source x-rays, and synchrotron x-rays, which have been employed for determining residual stress for many materials or component geometries [35], [28], [15], [13], [29], [10], [38], [21]. Neutrons are capable of penetrating samples that are on the order of centimeters and slits can be employed to measure the change in lattice spacings for crystals located at a particular internal point in a component [19], [24]. Neutron detection is slower than x-rays, however, and changes in lattice spacings typically are measured only along a few directions (scattering vectors). Laboratory sources produce x-rays that can be used to quantify residual stresses near the surface of a specimen in a reflection mode. Quantifying the residual stress field within a component using lab source $\mathrm{x}$-rays relies on removing layers of materials from the surface and investigating the newly exposed layers [39], [32], which introduces 
a significant uncertainty to the stress solution. Penetration through moderate thicknesses $(\mathrm{mm}-\mathrm{cm})$ of metallic samples combined with the advantages of high speed area detectors and short collection times have enabled a new generation of high energy synchrotron x-ray diffraction experiments [34], [48], [30], [49], [13], [33], [23], [5]. This large number of lattice strain measurements can be assembled into lattice strain pole figures [15], [46], [36], [33], which can be "inverted" to calculate the orientation-dependent strain and stress tensors within the polycrystalline diffraction volume [3], [47], [6]. The strain pole figure inversion method, which was employed in McNelis et al. [31] and is consistent with the method developed by Bernier et al. [6] for in situ loading experiments, is used in this work to make the connection between the measured lattice strains and the stress distribution over all crystal orientations within each diffraction volume.

\subsection{Lattice strain measurements using monochromatic x-ray powder diffraction}

Figure 1 shows a schematic of the transmission geometry employed to measure SPFs at an internal point within a sample. The sample and laboratory coordinate systems employed in this work were chosen carefully to illustrate the attributes of the experimental method and our results. While the incident monochromatic x-ray beam illuminates the entire volume of material in its beam path, a diffraction volume located at a particular point, $\mathbf{p}_{\mathrm{S}}$, inside the sample is isolated using an x-ray optical device referred to as conical slit [34]. For a particular $\{h k l\}$ and scattering vector, $\mathbf{q}$, all lattice planes in a diffraction volume and satisfying Bragg's law will diffract. The scattering vector is the bisector between the incident beam and the diffracted beams. The SPF information for the diffraction volume located at $\mathbf{p}_{\mathrm{S}}$ is obtained from the diffracted beam emanating from the diffraction volume located at $\mathbf{p}_{\mathbf{S}}$. The methodology for isolating a particular diffraction volume located inside the illuminated volume and the conical slit are described further in $\$ 2.2$ and $\$ 3$ respectively.

For an aggregate of crystals located at a particular $\mathbf{p}_{\mathrm{S}}$, the measured lattice strain, $\epsilon_{\mathbf{n n}}\left(\mathbf{p}_{\mathrm{S}}, \mathbf{q}\right)$, in those crystals having a particular family of planes ( $\{h k l\})$ with unit vector $\mathbf{n}$ parallel to a specific $\mathbf{q}$ is obtained using

$$
\epsilon_{\mathbf{n n}}\left(\mathbf{p}_{\mathrm{S}}, \mathbf{q}\right)=\frac{d_{\mathbf{n}}\left(\mathbf{p}_{\mathrm{S}}, \mathbf{q}\right)-d_{\mathbf{n}}^{0}}{d_{\mathbf{n}}^{0}}
$$

In this equation, $d_{\mathbf{n}}\left(\mathbf{p}_{\mathrm{S}}, \mathbf{q}\right)$ is the measured plane spacing for the $\{h k l\}$ located at $\mathbf{p}_{\mathrm{S}}$ with its $\mathbf{n}$ parallel to $\mathbf{q}$, and $d_{\mathbf{n}}^{0}$ is the reference (unstrained) plane spacing of the $\{h \mathrm{kl}\}$ of interest, which is calculated using a set of strain-free lattice parameters for a given crystal structure.

For an experiment using monochromatic x-rays, Equations 1 and 2 can be combined as

$$
\epsilon_{\mathbf{n n}}\left(\mathbf{p}_{\mathrm{S}}, \mathbf{q}\right)=\frac{\sin \theta_{\mathbf{n}}^{0}}{\sin \theta_{\mathbf{n}}\left(\mathbf{p}_{\mathrm{S}}, \mathbf{q}\right)}-1
$$

In this equation, $\theta_{\mathbf{n}}\left(\mathbf{p}_{\mathrm{S}}, \mathbf{q}\right)$ and $\theta_{\mathbf{n}}^{0}$ are Bragg angles that correspond to the plane spacings, $d_{\mathbf{n}}\left(\mathbf{p}_{\mathrm{S}}, \mathbf{q}\right)$ and $d_{\mathbf{n}}^{0}$ respectively.

The diffracted beams shown in Figure 1 form a cone around the transmitted beam known as the Debye-Scherrer cone. The polycrystalline sample is located at the apex of the Debye-Scherrer cone. Furthermore, if multiple families of crystallographic planes are considered, the Debye-Scherrer cones, each corresponding to one specific family of crystallographic planes, share an apex and a common axis.

\subsection{Ray tracing method}

As an X-ray beam passes all the way through a sample, it interacts with every grain in its path and each crystal with a suitably oriented lattice plane family $(\{h k l\})$ will diffract. The collection of all crystals "participating" in a particular diffraction event are said to lie along a crystallographic fiber in orientation space [9]. For a particular $\mathbf{q}$ and $\{h k l\}$, the diffracted intensity (peak) containing the signal from one fiber is recorded on the detector and the lattice strain indicated by the shift of the peak is an average value from all crystals within the fiber ${ }^{1}$. To isolate the diffracted intensity from a particular region along the beam's path through the sample and to measure the lattice strain associated with that region

\footnotetext{
${ }^{1}$ In this section, we treat the incident and diffracted beams as lines (rays) that coincide with the averages of the real distributions. This simplifies the treatment but still applies to the general case.
} 


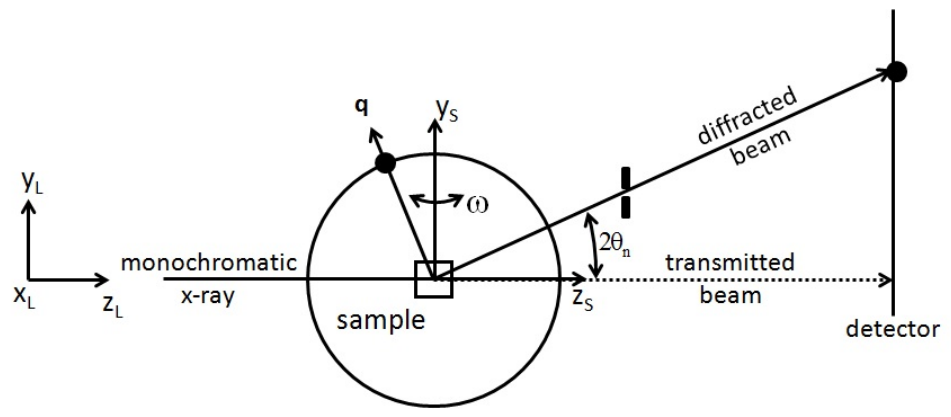

(a) The scattering vector, $\mathbf{q}$, is the bisector between the incident beam and the diffracted beam. The monochromatic x-ray beam illuminates the entire volume of material in its path and the illuminated volume of material participates in diffraction. A conical slit placed in the diffracted beam's path isolates a particular diffraction volume inside the illuminated volume as shown in Figure 1(b) The sample is rotated about $\mathrm{y}_{\mathrm{L}}$ by $\omega$ to interrogate many scattering vectors. The strain information for the isolated diffraction volume measured on the detector is plotted on the SPF surface (a unit sphere around the diffraction volume illustrated by the circle around the sample) at the intersection between the scattering vector and the SPF surface.

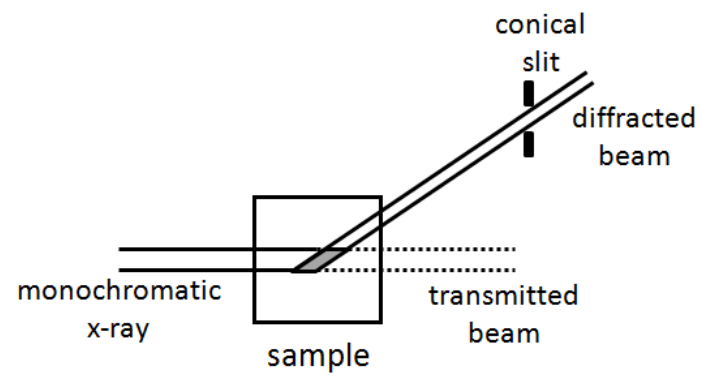

(b) A conical slit isolates a particular diffraction volume (gray area) inside the illuminated volume and only the diffracted beam emanating from the diffraction volume is recorded on the detector.

Figure 1: A schematic of the transmission geometry used for measuring SPFs in this work. 


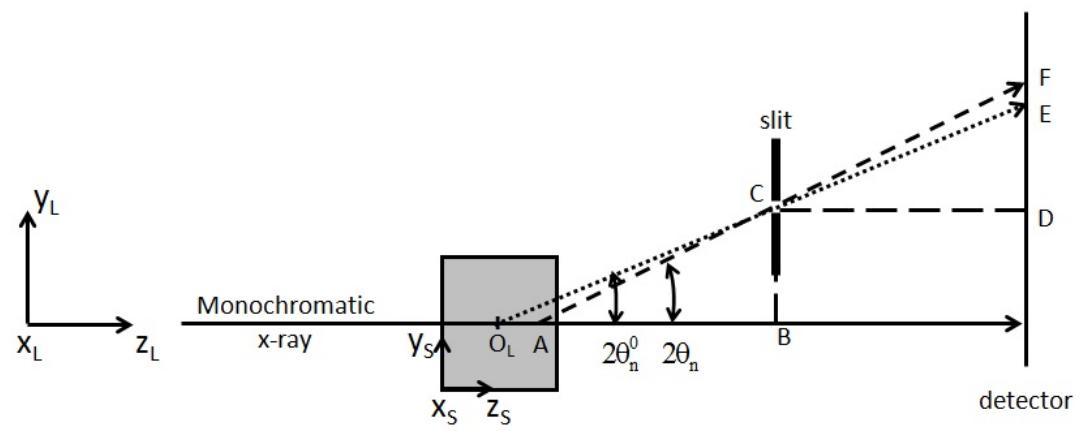

Figure 2: A schematic of the ray tracing method. The strained sample is placed such that $\mathbf{O}_{\mathbf{L}}$ and $\mathbf{O}_{\mathbf{S}}$ (centroid of the sample) are identical. The incident monochromatic X-ray beam illuminates the entire volume of material along $\mathrm{Z}_{\mathrm{S}}$. The slit is placed at $\mathbf{C}$. The line between $\mathbf{O}_{\mathbf{L}}$ and $\mathbf{E}$ is the path of the diffracted beam if a thin unstrained sample is placed at $\mathbf{O}_{\mathbf{L}}$. The line between $\mathbf{A}$ and $\mathbf{F}$ is the path of the diffracted beam emanating from the strained diffraction volume located at $\mathbf{A}$.

for the scattering vector and $\{h k l\}$ of interest, a slit can be positioned between the sample and the detector. Using the geometry of the experiment and the elements of Bragg's law, we are able to systematically "step through" the illuminated volume and obtain lattice strain information as a function of position. Combined with a sample rotation, this allows us to non-destructively measure the SPFs from the individual regions (diffraction volumes) located inside the sample.

A slit is added to the transmission geometry as illustrated in Figures 1 and 2 to enable ray tracing. Given the wavelength of the incident monochromatic x-ray beam and considering an unstrained polycrystalline aggregate, $\theta_{\mathbf{n}}^{0}$ is a known quantity for a particular $\{h k l\}$ of the crystals that constitute the aggregate. For an arbitrary point along the incident beam denoted as $\mathbf{O}_{\mathbf{L}}$, a slit is placed such that $\angle \mathrm{CO}_{\mathbf{L}} \mathrm{B}$ is equal to $2 \theta_{\mathbf{n}}^{0}$. The point $\mathbf{O}_{\mathbf{L}}$ is coincident with the apex of the Debye-Scherrer cones of the aggregate.

Given this construct, a thick polycrystalline aggregate is placed such that the centroid of the aggregate, $\mathbf{O}_{\mathbf{S}}$, is coincident with $\mathbf{O}_{\mathbf{L}}$. The line between $\mathbf{O}_{\mathbf{L}}$ and $\mathbf{E}$ is the diffraction beam path when an infinitesimally thin unstrained sample is placed at $\mathbf{O}_{\mathbf{L}}$. The line between $\mathbf{A}$ and $\mathbf{F}$ is the diffracted beam path originating from the diffraction volume located at $\mathbf{A}$. The difference between $\angle F A B$ and $\angle E O_{L} B$ is due to lattice strain at $\mathbf{A}$. Here, the lattice strain at $\mathbf{A}$ is assumed to be negative and thus $\mathbf{A}$ is downstream of $\mathbf{O}_{\mathbf{L}}$. While the entire thickness of the aggregate is illuminated, only a small portion of the illuminated volume whose strain satisfies both the Bragg diffraction condition and the geometric condition imposed by the conical slit contribute to the diffraction peak recorded at F. Thus, the goal of the ray tracing is to find the location of $\mathbf{A}$ with respect to $\mathbf{O}_{\mathbf{L}}$ (which is coincident with $\mathbf{O}_{\mathbf{S}}$ in this case) given a measurement at $\mathbf{F}^{2}$

Taking advantage of angular relationships and similar triangles shown in Figure 2 the following geometric relationships can be established:

$$
\tan 2 \theta_{\mathbf{n}}^{0}=\frac{|\mathrm{CB}|}{\left|\mathrm{O}_{\mathrm{L}} \mathrm{B}\right|}=\frac{|\mathrm{ED}|}{|\mathrm{CD}|}
$$

where the length of a line segment is denoted by vertical bars. For example, $|\mathrm{CB}|$ is the length of the line segment between point $\mathbf{C}$ and point $\mathbf{B}$. Similarly, the geometric relationships for the diffracted beam originating from $\mathbf{A}$ are

$$
\tan 2 \theta_{\mathbf{n}}(\mathbf{A}, \mathbf{q})=\frac{|\mathrm{CB}|}{|\mathrm{AB}|}=\frac{|\mathrm{FD}|}{|\mathrm{CD}|}
$$

\footnotetext{
${ }^{2}$ In other words, any point on the detector is mapped through the slit to exactly one point along $\mathrm{z}_{\mathrm{L}}$.
} 
Equations 4 and 5 can be rearranged to obtain the following

$$
\left|\mathrm{O}_{\mathrm{L}} \mathrm{A}\right|=\left|\mathrm{O}_{\mathrm{L}} \mathrm{B}\right| \frac{|\mathrm{FE}|}{|\mathrm{FD}|}
$$

where $|\mathrm{FE}|$ is a measured quantity, $\left|\mathrm{O}_{\mathrm{L}} \mathrm{B}\right|=\left|\mathrm{O}_{\mathrm{L}} \mathrm{C}\right| \cos 2 \theta_{\mathbf{n}}^{0}$, and $|\mathrm{ED}|+|\mathrm{FE}|=|\mathrm{FD}|$ where $|\mathrm{ED}|=|\mathrm{CE}| \sin 2 \theta_{\mathbf{n}}^{0}$. In this construct, $\mathbf{O}_{\mathbf{L}}$ and $\mathbf{O}_{\mathbf{S}}$ are coincident and the position of $\mathbf{A}$ with respect to $\mathbf{O}_{\mathbf{S}}$ can be computed using Equation 6 . The lattice strain at $\mathbf{A}$ can be computed using $\angle \mathrm{FAB}, \angle \mathrm{EO}_{\mathrm{L}} \mathrm{B}$, and Equation 3 .

Finally, if the sample is placed in the beam path such that $\mathbf{O}_{\mathbf{L}}$ is no longer coincident with $\mathbf{O}_{\mathbf{S}}$, the diffraction peak from a diffraction volume located elsewhere in the illuminated volume can be recorded. Equations 4 -6 will still hold, but now $\left|\mathrm{O}_{\mathrm{L}} \mathrm{A}\right|=\left|\mathrm{O}_{\mathbf{L}} \mathrm{O}_{S}\right|+\left|\mathrm{O}_{S} \mathrm{~A}\right|$ where $\left|\mathrm{O}_{\mathbf{L}} \mathrm{O}_{\mathbf{S}}\right|$ is the known offset between $\mathbf{O}_{\mathbf{L}}$ and $\mathbf{O}_{\mathbf{S}}$. This implies that if the sample is systematically translated along the incident beam path while a series of diffraction measurements are made, the lattice strains for distinct diffraction volumes together with their locations inside the illuminated volume can be obtained. Similar relationships can be derived for the case where the lattice strain at $\mathbf{A}$ is positive. It is worthwhile to note that the $\mathrm{x}_{\mathrm{S}}$ and $\mathrm{y}_{\mathrm{S}}$ coordinates of $\mathbf{A}$ are simply the $\mathrm{x}_{\mathrm{S}}$ and $\mathrm{y}_{\mathrm{S}}$ coordinates of the illuminated volume. On the other hand, the $\mathrm{z}_{\mathrm{S}}$ coordinate of $\mathbf{A}$ is determined through ray tracing. We denote the $\mathrm{z}_{\mathrm{S}}$ coordinate of a diffraction volume determined through ray tracing as $\mathrm{z}_{\mathrm{S}}^{\star}$.

\section{Key features of the conical slits}

A set of slits capable of interrogating the full Debye-Scherrer cones for multiple $\{h k l\}$ s simultaneously has been designed to take advantage of the ray tracing method. The set of conical slits used in this work is illustrated in Figure 3. It is a standard equipment at the 1-ID-C beam line of the Advanced Photon Source where this experimental work was performed. The readers are referred to Lienert et al. [27] and Nielsen et al. [34] for a more detailed description of the conical slits. The following are the key features of the conical slits used in this work.

- A set of concentric rings each with a different radius are assembled and held together by the four spokes. The channels created by the mismatch in the ring radii work as slits.

- The conical slits are constructed assuming that the distance between $\mathbf{O}_{\mathbf{L}}$ and $\mathbf{B}$ is $50 \mathrm{~mm}$.

- The radii of the slits are selected such that multiple $\{h k l\}$ s and scattering vectors can be interrogated simultaneously for materials with face-centered cubic (FCC) crystal symmetry. The ideal slit radii and the crystallographic planes interrogated by the slits are listed in Table 1

- Because the distance between $\mathbf{O}_{\mathbf{L}}$ and $\mathbf{B}$ is fixed, the wavelength of the monochromatic x-ray beam is changed to accommodate different materials.

- The width of the slits are $20 \mu \mathrm{m}$.

- The concentric rings are $2 \mathrm{~mm}$ thick along the $\mathrm{z}_{\mathrm{L}}$-direction and are made of tungsten carbide to attenuate the diffracted beams that do not satisfy the geometric condition imposed by the conical slits.

- Because the slits have the same width, the spatial resolution along the $\mathrm{z}_{\mathrm{L}}$-direction depends on $\{h k l\}$. The $\{h k l\}$-dependent spatial resolutions are tabulated in Table 1

\section{Experimental setup and method}

\subsection{Material and sample}

The material used in this work is low-solvus high-refractory (LSHR) Ni-base superalloy developed for high temperature applications [14]. Like most nickel-base alloys, LSHR consists of a FCC matrix solid solution $(\gamma)$ interspersed 


\begin{tabular}{cccc} 
& $\{h k l\}$ & Distance between B and $C_{i}(\mathrm{~mm})$ & Spatial Resolution $(\mathrm{mm})$ \\
\hline \hline $\mathrm{BC}_{1}$ & 111 & 4.838 & 0.208 \\
$\mathrm{BC}_{2}$ & 200 & 5.597 & 0.180 \\
$\mathrm{BC}_{3}$ & 220 & 7.947 & 0.127 \\
$\mathrm{BC}_{4}$ & 222 & 9.779 & 0.104 \\
$\mathrm{BC}_{5}$ & 331 & 12.408 & 0.083 \\
$\mathrm{BC}_{6}$ & 422 & 14.0299 & 0.074
\end{tabular}

Table 1: Geometric characteristics of the conical slits used in this work.
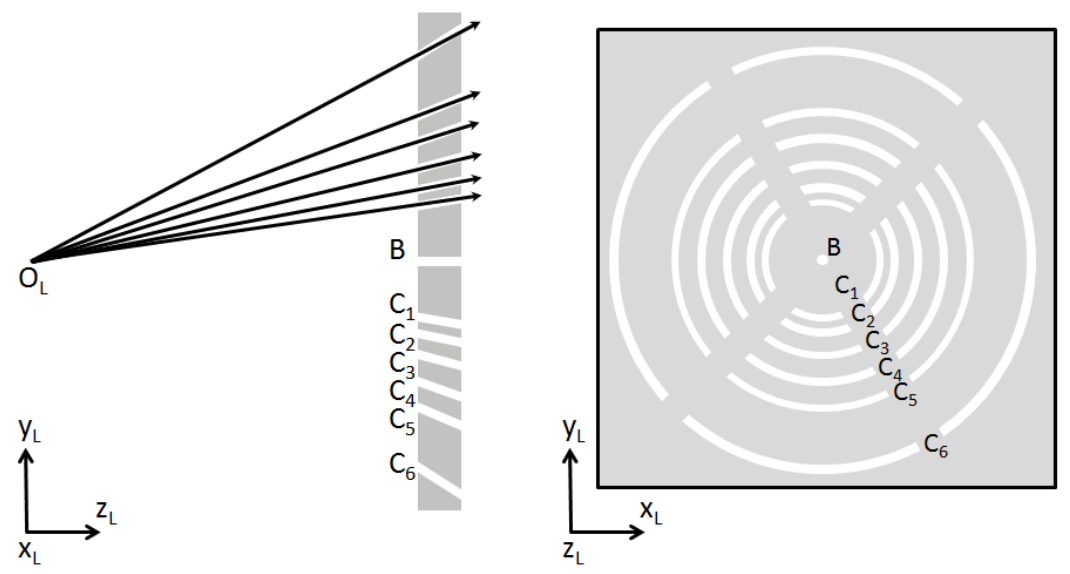

Figure 3: Illustration of the conical slit assembly used in this work viewed from two directions. A set of concentric rings each with a different radius are assembled and held together by the four spokes. The channels created by the mismatch in the ring radii work as slits to allow diffracted beam to pass through. Here, $\mathbf{O}_{\mathbf{L}}$ is the apex of the DebyeScherrer cones, $\mathbf{B}$ is the center hole that allows the incident x-rays to pass through for alignment purposes, and $\mathbf{C}_{\mathbf{i}} \mathbf{s}$ are the locations of the slits. 


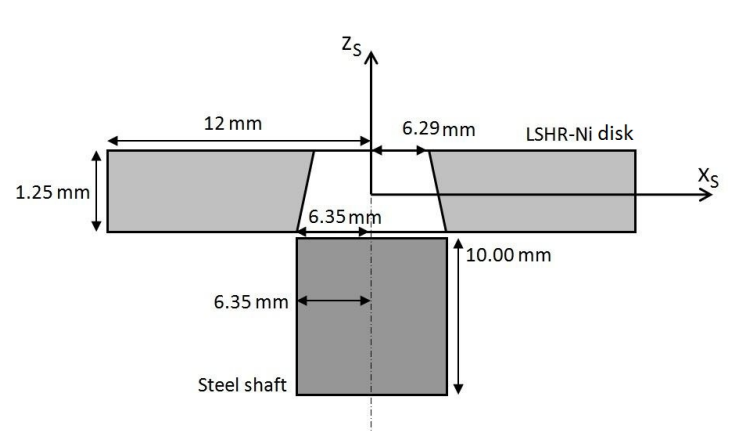

(a) A schematic of the disk and the shaft of the sample before assembly.

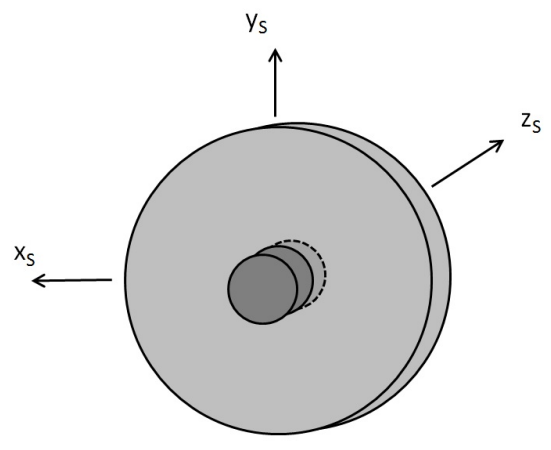

(b) A schematic of assembled sample.

Figure 4: A schematic of the disk and the shaft of the sample before and after assembly. The drawings are not to scale.

with coherent $\gamma^{\prime}$ precipitates, which serve as the primary strengthening feature [40], [37]. Even though the precipitate volume fraction can be quite high, the coherency of the $\gamma^{\prime}$ with the $\gamma$ phase allows Ni-base superalloys to be treated as a single FCC phase structurally and crystallographically [44] albeit with small superlattice peaks appearing within the spectrum. From this point forward, we will refer to the material simply as LSHR. The crystallographic orientation distribution of the material is close to uniform [31]. The macroscopic yield strength and Young's modulus of the LSHR are $1.11 \mathrm{GPa}$ and $190 \mathrm{GPa}$ respectively. Using a thin piece of LSHR extracted from the bulk, a powder pattern was obtained to determine its lattice parameter in its strain-free state $(0.3593 \mathrm{~nm})$ [11]. This lattice parameter was used to compute the reference lattice plane spacings for the crystallographic planes that were interrogated in this work. The average grain size of the material is approximately $3 \mu \mathrm{m}$ [31].

An interference fit geometry was employed to fabricate a sample containing a 3D residual stress field that varies throughout the body. Unlike the geometry employed by McNelis et al. [31], the inner diameter of the disk was tapered along the disk thickness to generate a 3D residual stress field in the disk. Figure 4(a) shows a schematic of the sample with its dimensions before assembly.

To assemble the interference fit sample, the disk was heated to approximately $800^{\circ} \mathrm{C}$ in air while the shaft remained at room temperature. When the disk reached the desired temperature, it was removed from the furnace. The disk and the shaft were assembled as shown in Figure 4(a) and the sample was allowed to cool. The sample coordinate system, as depicted in Figure 4(b), is such that the $x_{S}$ and $y_{S}$ are in the plane of the disk and $z_{S}$ is such that the inner radius of the disk becomes smaller with increasing $\mathrm{z}_{\mathrm{S}}$.

Since the inner radius of the disk before assembly changes with the disk thickness, the stresses in the disk following the shrink fitting process are not uniform through the thickness of the disk. A 3D elastic finite element model of the sample geometry was used to obtain the approximate stress distribution that could be anticipated in the disk after assembly. The stress distribution from the 3D elastic FE model (Figure 5) varies with $\mathrm{x}_{\mathrm{S}}$ and $\mathrm{y}_{\mathrm{S}}$, similar to the conventional interference fit. The magnitudes of the stress components are the largest where the interference is the largest and the magnitudes of the stress components are the smallest at the outer radial position. The distribution also varies along $\mathrm{z}_{\mathrm{S}}$ due to the tapered inner radius of the disk. It is important to note that these results only serve as a qualitative guide to interpret the stress distribution obtain via the SPFs and the bi-scale optimization scheme.

If the assembly of the sample is perfect and the magnitude of the interference is well-known, the stress field on the $\mathrm{x}_{\mathrm{S}}-\mathrm{z}_{\mathrm{S}}$ plane are identical to that on the $\mathrm{y}_{\mathrm{S}} \mathrm{z}_{\mathrm{S}}$ plane or any other slice through the disk in a polar coordinate system. However, in a real sample where the assembly is imperfect and the magnitude of the interference is not well-known, this is not the case and we choose to represent the stresses in the Cartesian coordinate system to illustrate the variability that we were able to capture. The Cartesian coordinate system that we use is also consistent with the coordinate system employed in representing the strain pole figures; this enables us to compare the trends in the strain pole figures to the 3D stress distribution obtained by using the strain pole figures and the bi-scale optimization scheme. 

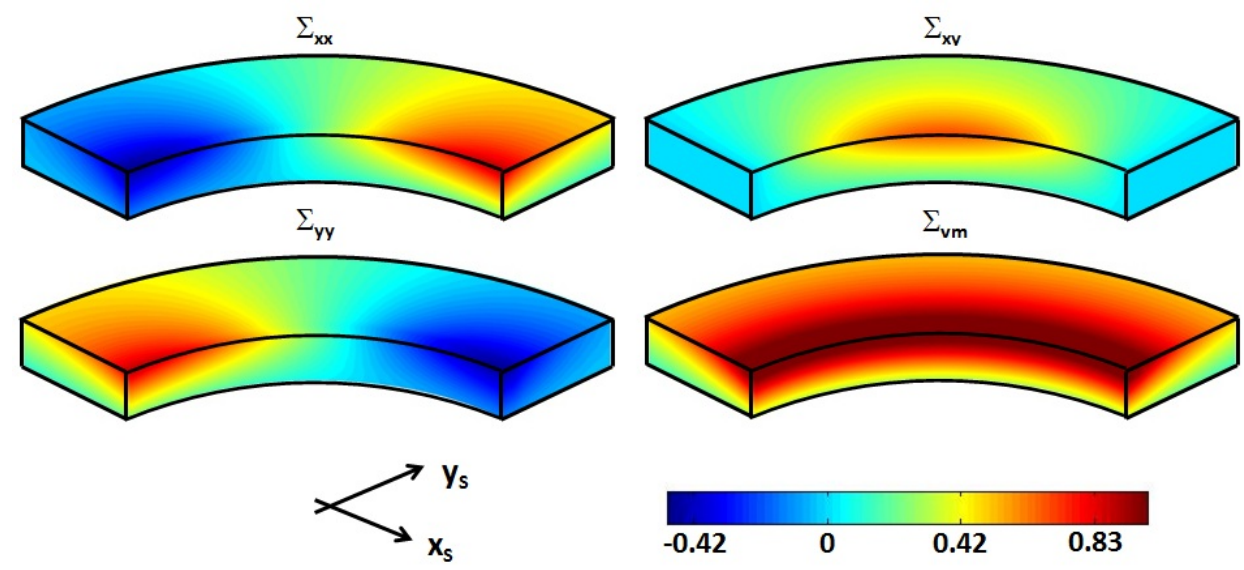

Figure 5: An approximation of the stress field in the interference fit sample obtained by a 3D elastic FE simulation of a quarter of the disk. The stress field is normalized by the maximum of the second invariant of the stress to show the anticipated trends of the stress field. Two normal components and one shear component of the stress, denoted by $\Sigma_{\mathrm{xx}}$, $\Sigma_{\mathrm{yy}}$, and $\Sigma_{\mathrm{xy}}$ respectively, are shown. The values of the other stress components are negligible. The second invariant stress denoted by $\Sigma_{\mathrm{vm}}$ is also shown.

\subsection{X-ray properties}

The monochromatic $\mathrm{x}$-ray beam used in this work was focused using the $\mathrm{x}$-ray optics described in [41] and the final beam size was $50 \mu \mathrm{m}$ along the $\mathrm{y}_{\mathrm{L}}$ direction and $50 \mu \mathrm{m}$ along the $\mathrm{x}_{\mathrm{L}}$. The wavelength of the beam used to measure the lattice strains in the interference fit sample was $0.02000 \mathrm{~nm}(61.99 \mathrm{keV})$. This wavelength was chosen so that the diffracted beams from an unstrained LSHR sample would pass through the conical slits when it is placed at $\mathbf{O}_{\mathbf{L}}$.

\subsection{Data acquisition}

An amorphous Si area detector [26] was used to collect the diffraction data. The area detector was placed such that only the $\{111\},\{200\},\{220\},\{222\}$ rings could be recorded. From these, only the $\{111\},\{200\}$, and $\{220\}$ SPFs were generated as the intensity of the $\{222\}$ peak decreased significantly at large $\omega$ angles.

Figure 6(a) shows a map of the illuminated volume locations on the disk. Assuming sample symmetry, only a quarter of the disk was examined. Within this quadrant, four equally spaced angular positions, denoted by $\alpha_{\mathrm{S}}$, were interrogated. For each $\alpha_{\mathrm{S}}$ position, six radial positions, denoted by $\mathrm{r}_{\mathrm{S}}$, were interrogated. As alluded in 2.2 , these are the $\mathrm{x}_{\mathrm{S}}$ and $\mathrm{y}_{\mathrm{S}}$ coordinates of the diffraction volumes where the SPFs were measured. The polar coordinates of the illuminated volume can be converted to the Cartesian coordinates $\left(\mathrm{x}_{\mathrm{s}}, \mathrm{y}_{\mathrm{s}}\right)$ and these are used interchangeably.

To obtain the lattice strain information and corresponding $\mathrm{z}_{\mathrm{S}}$ position determined by ray tracing $\left(\mathrm{z}_{\mathrm{S}}^{\star}\right)$ for all diffraction volumes located inside the illuminated volume located at a particular $\left(\alpha_{\mathrm{S}}, \mathrm{r}_{\mathrm{S}}\right)$, the sample was translated along $\mathrm{z}_{\mathrm{L}}$ and a series of diffraction data were recorded. For a particular $\left(\alpha_{\mathrm{S}}, \mathrm{r}_{\mathrm{S}}\right)$, the diffraction data were obtained by the following procedure.

1. Using $\mathrm{x}_{\mathrm{L}}-\mathrm{y}_{\mathrm{L}}$ translation, move the sample to illuminate the aggregate of crystals located at $\left(\alpha_{\mathrm{S}}^{\mathrm{SPF}}, \mathrm{r}_{\mathrm{S}}^{\mathrm{SPF}}\right)$.

2. Using a rotation stage, rotate the sample to the desired $\omega$.

3. Systematically translate the sample along $\mathrm{z}_{\mathrm{S}}$ between a set interval and acquire a series of diffraction images.

4. Repeat the previous steps for all $\omega$ positions between $-60^{\circ}$ and $60^{\circ}$ at $5^{\circ}$ intervals where $\omega=0^{\circ}$ is normal incidence.

At normal incidence $\left(\omega=0^{\circ}\right)$, the distance between $\mathbf{O}_{\mathbf{L}}$ and $\mathbf{O}_{\mathbf{S}}$ ranged between $\pm 0.95 \mathrm{~mm}$ and 19 steps were taken between the limits. At the limits of the translation along $\mathrm{z}_{\mathrm{S}}$, the diffraction images exhibit insufficient intensity 

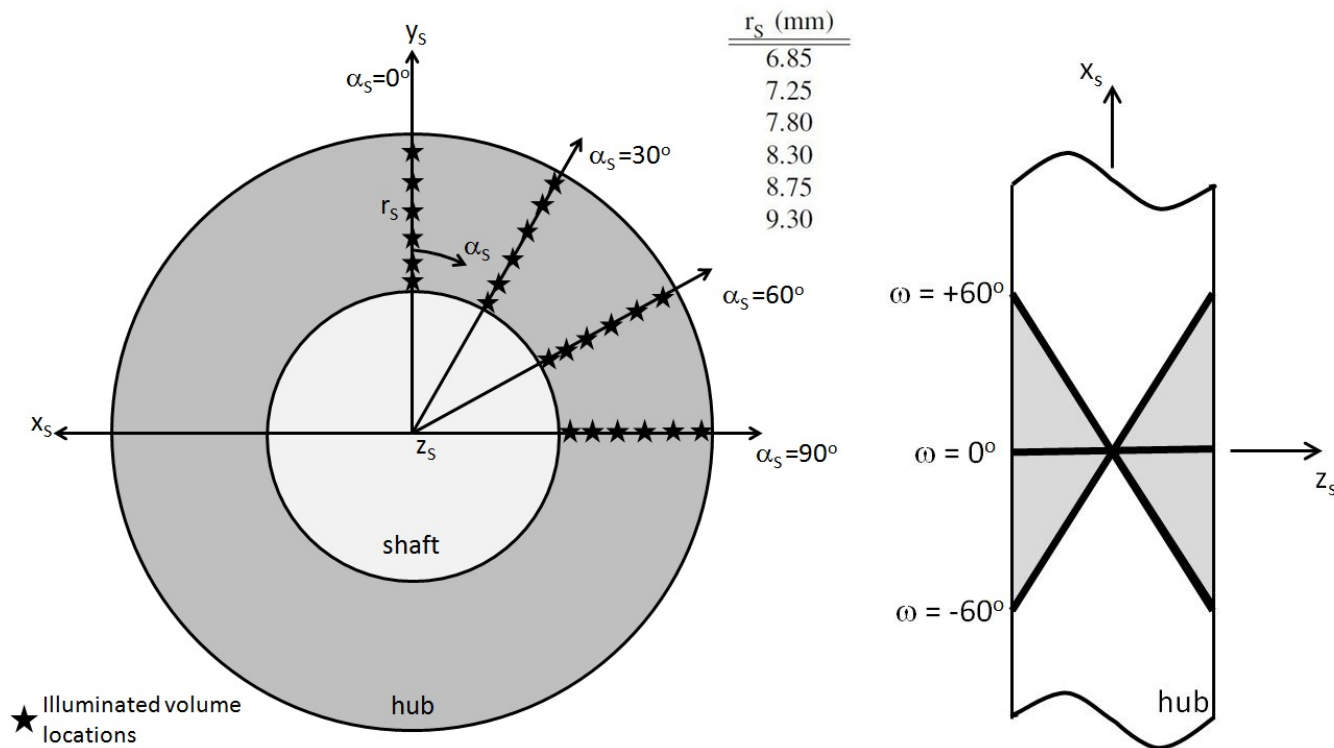

(a) A map of the illuminated volume location on the disk where diffraction data were recorded through the thickness of the disk. The stars indicate the locations of the illuminated volumes. The angular and radial positions of the illuminated volume locations are denoted as $\alpha_{\mathrm{S}}$ and $\mathrm{r}_{\mathrm{S}}$ respectively. For each $\alpha_{\mathrm{S}}$, six illuminated volumes are interrogated radially and the values of $r_{S}$ are listed in the table.

(b) A schematic of the effective shape of the illuminated volume. The area shaded in gray is the shape of the illuminated volume due to the $\omega$ rotation.

Figure 6: A map of the illuminated volume locations and the effective shape of the illuminated volume. 


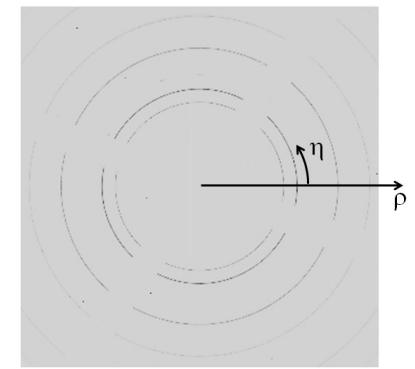

(a) A raw diffraction image recorded when $\omega=0^{\circ}$, $\alpha_{\mathrm{S}}=0^{\circ}, \quad \mathrm{r}_{\mathrm{S}}=8.75 \mathrm{~mm}$, and $\mathrm{O}_{\mathrm{L}}=\mathrm{O}_{\mathrm{S}}$. A polar coordinate system defined by the radius, $\rho$, and the azimuthal angle, $\eta$, is established to transform the raw diffraction image into intensity vs. $\rho$ spectra around $\eta$.

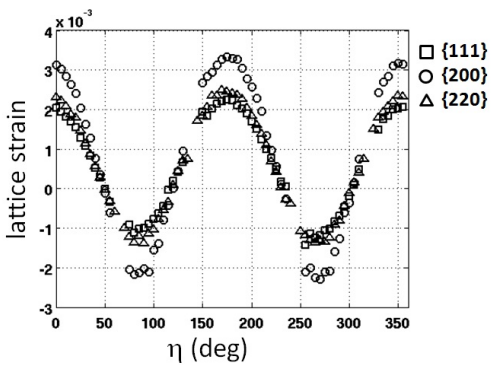

(b) The measured lattice strains vs. $\eta$ obtained from the example diffraction data shown in Figure 7(a) The lattice strains for four crystallographic planes $\{111\}$, $\{200\}$, and $\{220\}$ measured around $\eta$ are plotted.

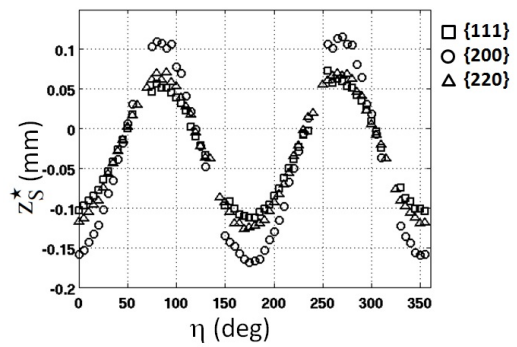

(c) The locations of the diffraction volumes $\left(\mathrm{z}_{\mathrm{S}}^{\star}\right)$ corresponding to the lattice strains shown in Figure $7(\mathrm{~b})$

Figure 7: An illustration of the data reduction method used in this work.

to render useful peaks. Therefore, the range of translation was deemed sufficient to interrogate the full thickness of the sample. For the non-zero values of $\omega$, the effective thickness of the sample increases with $\omega$. Thus, the sample translation step size was adjusted to reflect this change and to interrogate the individual diffraction volumes in the elongated effective thickness.

Due to the $\omega$ rotation, the volume of the material illuminated through the thickness of the disk has an hourglass shape as illustrated in Figure 6(a) The conical slits described in $\$ 3$ isolate individual diffraction volumes in this hourglass shape along the $\mathrm{z}_{\mathrm{S}}$ direction.

Using the data acquisition procedure, a total of 11,400 diffraction images were recorded (25 steps in $\omega \times 19$ steps in $\mathrm{z}_{\mathrm{S}} \times 24$ illuminated volumes). These images were analyzed to obtain $\mathrm{z}_{\mathrm{S}}^{\star}$ and lattice strain information for $\{111\}$, $\{200\}$, and $\{220\}$ peaks.

\section{Extracting the SPFs from diffraction data}

\subsection{Determination of lattice strains and diffraction volume coordinates from diffraction images}

Figure 7 summarizes the procedure used to reduce the diffraction data into lattice strains. An example of a raw diffraction image is shown in Figure 7(a). This image was recorded when $\omega=0^{\circ}, \alpha_{\mathrm{S}}=0^{\circ}, \mathrm{r}_{\mathrm{S}}=7.80 \mathrm{~mm}$, and the centroid of the disk along $\mathbf{z}_{\mathbf{S}}$ is at $\mathbf{O}_{\mathbf{L}}$. The intensities of the $\{222\}$ peaks were comparable to the inner peaks which is typically not the case at other $\omega$ positions.

Using a polar coordinate system defined by the radius from the center of the Debye-Scherrer rings, $\rho$, and the azimuthal angle, $\eta$, and appropriate experimental geometry and detector distortion parameters, the image was transformed to produce a set of intensity vs. radius spectra for all azimuthal angles [33]. The $\{111\},\{200\}$, and $\{220\}$ peaks were fitted using a pseudo-Voigt peak profile function to determine their peak positions at each azimuthal angle.

The intensity of the peak and the goodness of fit for the peak fitting process were examined to determine whether a peak would yield lattice strain and diffraction volume location information with low uncertainty. When a peak had suitable intensity and goodness of fit, its peak position and Equation 3 were used to compute the lattice strain. The location of the diffraction volume inside the disk where the peak emanated from $\left(\mathrm{O}_{\mathrm{S}} \mathrm{A}\right.$ or $\left.\mathrm{z}_{\mathrm{S}}^{\star}\right)$ was determined using 
the peak position and the ray tracing method. Thus, the location of the diffraction volume within the disk where a particular peak emanated from is $\left(\mathrm{x}_{\mathrm{S}}, \mathrm{y}_{\mathrm{S}}, \mathrm{z}_{\mathrm{S}}^{\star}\right)$.

Figure $7(\mathrm{~b})$ shows the lattice strains around $\eta$ for the $\{111\},\{200\}$, and $\{220\}$ peaks recorded in the diffraction image shown in Figure $7(\mathrm{a})$. Figure $7(\mathrm{c})$ shows the corresponding location of the diffraction volumes for the $\{111\}$, $\{200\}$, and $\{220\}$ peaks. This figure illustrates the challenges associated with analyzing the data obtained using the set of conical slits. The peaks in the example diffraction data are actually emanating from different locations inside the sample. For example, the peaks that show tensile lattice strains are originating from locations inside the sample that are approximately $-150 \mu \mathrm{m}$ away from $\mathrm{O}_{\mathrm{S}}$ while the peaks that show compressive lattice strains are originating from locations inside the sample that are approximately $100 \mu \mathrm{m}$ away from $\mathrm{O}_{\mathrm{S}}$. This is a key difference between the lattice strains measured in this work and the lattice strains measured using a conventional SPF experiment described in Miller et al.[33].

Because this image was taken at $\alpha_{\mathrm{S}}=0^{\circ}$, the $\mathrm{x}_{\mathrm{S}}$-direction corresponds to $\eta=0^{\circ}$ and $\eta=180^{\circ}$. Similarly, the ys-direction corresponds to $\eta=90^{\circ}$ and $\eta=270^{\circ}$. Along $\alpha_{\mathrm{S}}=0^{\circ}$, the xx-component (the hoop component) of the residual stress is expected to be tensile and the yy-component (the radial component) of the residual stress is expected to be compressive. The lattice strains shown in Figure 7(b) are consistent with the anticipated residual stress. The lattice strains for all three crystallographic planes are tensile at $\eta=0^{\circ}$ and $\eta=180^{\circ}$ and compressive at $\eta=90^{\circ}$ and $\eta=270^{\circ}$; they are also close to zero near $\eta=45^{\circ}, \eta=135^{\circ}, \eta=225^{\circ}$, and $\eta=315^{\circ}$.

Considering the dimensions and the geometry associated with the conical slits and the three reflections that produced SPFs, the average through-thickness resolution of the conical slits, denoted as $z_{\mathrm{S}}^{\text {res }}$, is approximately $170 \mu \mathrm{m}$.

The lattice strain measurement uncertainty is determined by considering the systematic aberration of the detector and the geometry of the experimental setup [26]. Using the uncertainty estimation methodology proposed by Schuren et al. [42], the lattice strain measurement uncertainty is on average $5 \times 10^{-5}$ for the three $\{h k l\}$ s interrogated.

\subsection{Building The SPFs}

The complete set of diffraction images recorded at a particular $\left(\alpha_{\mathrm{S}}, \mathrm{r}_{\mathrm{S}}\right)$ or $\left(\mathrm{x}_{\mathrm{S}}, \mathrm{y}_{\mathrm{S}}\right)$ through $\mathrm{z}_{\mathrm{S}}$ translation and $\omega$ rotation were converted to an array of $\epsilon_{\mathbf{n n}}\left(\mathbf{p}_{S}^{\star}, \mathbf{q}\right)$ values for the $\{111\},\{200\}$, and $\{220\}$ peaks and all possible scattering vectors interrogated by $\omega$ rotation. Here, $\mathbf{p}_{S}^{\star}$ is equal to $\left(\mathrm{x}_{\mathrm{S}}, \mathrm{y}_{\mathrm{S}}, \mathrm{z}_{\mathrm{S}}^{\star}\right)$. This information must be binned such that the $\{111\},\{200\}$, and $\{220\}$ SPFs for a particular individual diffraction volume within the illuminated volume can be generated.

To regroup the lattice strain information, arbitrary intervals along $\mathrm{z}_{\mathrm{S}}$ are established by considering the average through-thickness resolution of the conical slits. The midpoints of the intervals are denoted as $z_{\mathrm{S}}^{\mathrm{SPF}}$ and neighboring $z_{S}^{S P F}$ are separated by $z_{S}^{\text {res. }}$. The values of $z_{S}^{\mathrm{SPF}}$ are $-0.595 \mathrm{~mm},-0.425 \mathrm{~mm},-0.255 \mathrm{~mm},-0.085 \mathrm{~mm}, 0.085 \mathrm{~mm}, 0.255$ $\mathrm{mm}, 0.425 \mathrm{~mm}$, and $0.595 \mathrm{~mm}$. For a given value of $\mathrm{z}_{\mathrm{S}}^{\mathrm{SPF}}$ and $\{h \mathrm{kl}\}$, all lattice strain information whose $\mathrm{z}_{\mathrm{S}}^{\star}$ values fall in between $z_{S}^{S P F}-z_{S}^{\text {res }} / 2$ and $z_{S}^{S P F}+z_{S}^{\text {res }} / 2$ contribute to the $S P F$ for the diffraction volume located at $\left(x_{S}^{S P F}, y_{S}^{S P F}, z_{S}^{S P F}\right)$.

Figure 8 shows some examples of the SPFs measured in this work. This figure shows the $\{111\}$ and $\{200\}$ SPFs from several locations along $\alpha_{\mathrm{S}}=0^{\circ}$ and $\alpha_{\mathrm{S}}=90^{\circ}$. It is important to note that for a particular $\mathrm{SPF}$ at $\left(\alpha_{\mathrm{S}}^{\mathrm{SPF}}, \mathrm{r}_{\mathrm{S}}^{\mathrm{SPF}}, \mathrm{z}_{\mathrm{S}}^{\mathrm{SPF}}\right)$, the lattice strain data that constitute the SPF are not necessarily originating from $\mathrm{z}_{\mathrm{S}}^{\mathrm{SPF}}$ as designated. They are from a range of $z_{S}^{\star}$ positions that fall in the interval. The areas on the SPFs near the $\mathrm{z}$-axis where there are no data reflect the $\omega$ rotation range. The empty strips on the SPFs are due to the spokes on the conical slit assembly (Figure 3). The SPFs presented in Figure 8 are a small subset of the full SPF data and are presented here to illustrate the experimental results.

It is worthwhile to note that the set of SPF data for a particular diffraction volume located at $\mathbf{p}_{S}^{\star}$ also contains information suitable for conventional $\sin ^{2} \Psi$ residual stress analysis. The relationship between the coordinate system employed in conventional $\sin ^{2} \Psi$ residual stress analysis $(\Psi$ and $\phi)$ and the angles used in this work $(\omega$ and $\eta)$ is illustrated in Figure 罒. Two values of $\omega$ are considered for illustration: $\omega=0^{\circ}$ (Figure 9(a) and $\omega \neq 0^{\circ}$ (Figure 9(b)). In both cases, the inclination angle, $\Psi$, is the angle between $\mathrm{z}_{\mathrm{S}}$ and a particular scattering vector. The angle between the plane defined by $\mathrm{z}_{\mathrm{S}}$ and the scattering vector and the $\mathrm{x}_{\mathrm{S}}-\mathrm{z}_{\mathrm{S}}$ plane is $\phi$. When $\omega=0^{\circ}, \phi$ and $\eta$ are identical. When $\omega \neq 0^{\circ}, \phi$ and $\eta$ are not identical.

\footnotetext{
${ }^{3}$ For a detailed treatment of the $\sin ^{2} \Psi$ analysis for the determination of residual stress at a point, readers are referred to works by Lu [28] or Hauk et al. [15].
} 


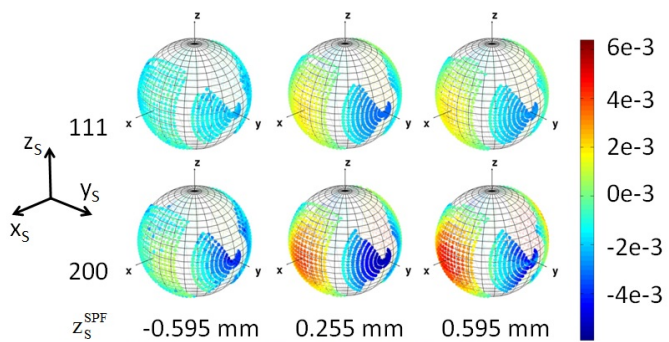

(a) The SPFs for $\{111\}$ and $\{200\}$ from several $z_{S}^{S P F}$ at $\mathrm{r}_{\mathrm{S}}=6.85 \mathrm{~mm}$ and $\alpha_{\mathrm{S}}=0^{\circ}$.

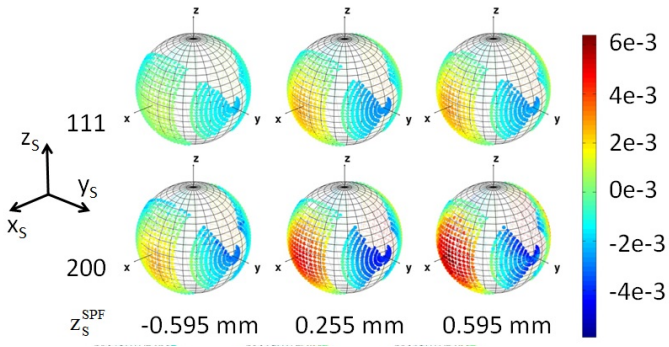

(c) The SPFs for $\{111\}$ and $\{200\}$ from several $z_{\mathrm{S}}^{\mathrm{SPF}}$ at $\mathrm{r}_{\mathrm{S}}=7.80 \mathrm{~mm}$ and $\alpha_{\mathrm{S}}=0^{\circ}$.

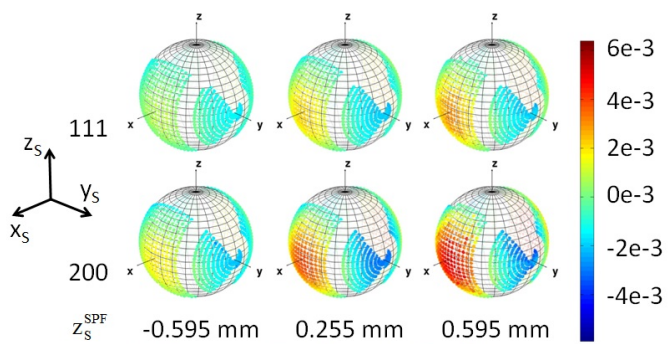

(e) The SPFs for $\{111\}$ and $\{200\}$ from several $z_{S}^{S P F}$ at $\mathrm{r}_{\mathrm{S}}=9.30 \mathrm{~mm}$ and $\alpha_{\mathrm{S}}=0^{\circ}$.

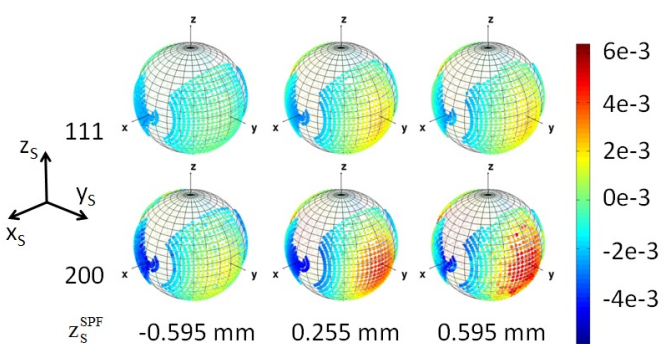

(b) The SPFs for $\{111\}$ and $\{200\}$ from several $z_{\mathrm{S}}^{\mathrm{SPF}}$ at $\mathrm{r}_{\mathrm{S}}=6.85 \mathrm{~mm}$ and $\alpha_{\mathrm{S}}=90^{\circ}$.

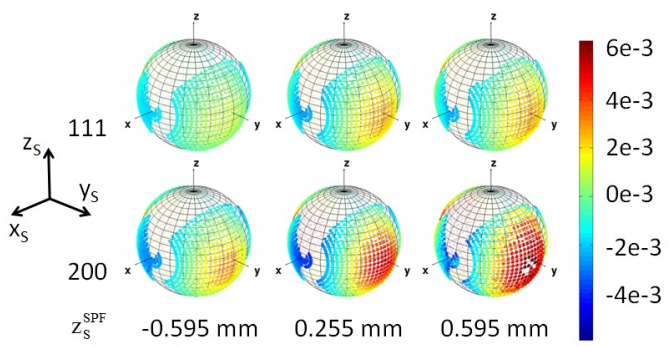

(d) The SPFs for $\{111\}$ and $\{200\}$ from several $z_{S}^{S P F}$ at $\mathrm{r}_{\mathrm{S}}=7.80 \mathrm{~mm}$ and $\alpha_{\mathrm{S}}=90^{\circ}$.

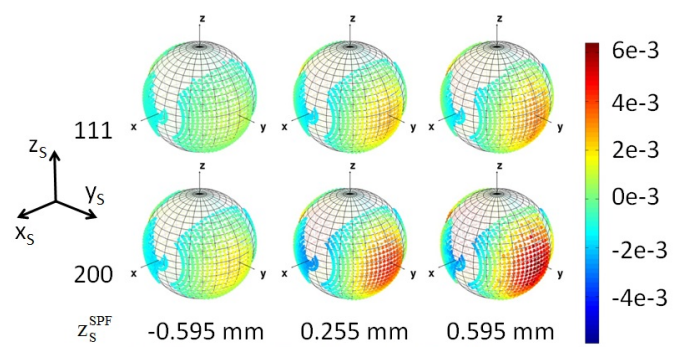

(f) The SPFs for $\{111\}$ and $\{200\}$ from several $z_{\mathrm{S}}^{\mathrm{SPF}}$ at $\mathrm{r}_{\mathrm{S}}=9.30 \mathrm{~mm}$ and $\alpha_{\mathrm{S}}=90^{\circ}$.

Figure 8: A subset of SPFs generated in this work. Figures $8(\mathrm{a}), 8(\mathrm{a})$, and $8(\mathrm{e})$ show a set of SPFs from three $\mathrm{r}_{\mathrm{S}}$ positions along $\alpha_{\mathrm{S}}=0^{\circ}$. Figures $8(\mathrm{~b}), 8(\mathrm{~d})$ and $8(\mathrm{f})$ show a set of SPFs from three $\mathrm{r}_{\mathrm{S}}$ positions along $\alpha_{\mathrm{S}}=90^{\circ}$. 


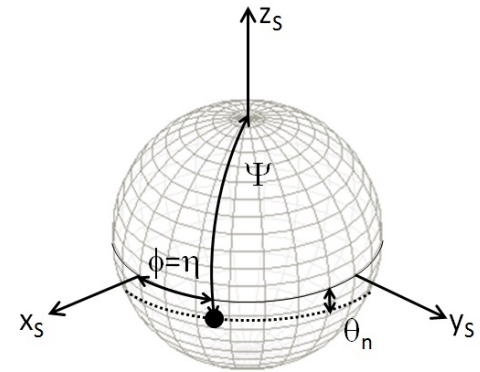

(a) For a particular scattering vector interrogated when $\omega=0^{\circ}$ (black dot), $\eta$ is identical to $\phi$.

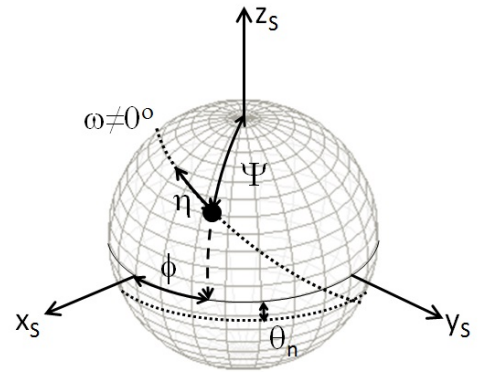

(b) For a particular scattering vector interrogated when $\omega \neq 0^{\circ}$ (black dot), $\eta$ is not identical to $\phi$.

Figure 9: The relationship between the angular convention used in $\sin ^{2} \Psi$ residual stress measurement technique ( $\Psi$ and $\phi)$ and the angles used in this work $(\omega$ and $\eta)$ is illustrated. The angle between $\mathrm{z}_{\mathrm{S}}$ and the scattering vector is $\Psi$. The angle between the plane defined by $\mathrm{z}_{\mathrm{S}}$ and the scattering vector and the $\mathrm{x}_{\mathrm{S}}-\mathrm{z}_{\mathrm{S}}$ plane is $\phi$.

\section{Determining the 3D residual stress field using the bi-scale optimization scheme}

A bi-scale optimization scheme is called upon to estimate a residual stress field in the sample based on the lattice strain data collected for the array of diffraction volumes. The method employed here was developed specifically for this purpose. In this section, we summarize the key features of the method and refer the reader to McNelis et al. [31] and Demir et al. [12] for a thorough exposition. An overview of the method is presented in Figure 10 which serves as a guide for the summary.

The overall objective is to determine a macroscopic, or continuum scale, stress distribution, designated as $\Sigma^{h}(\boldsymbol{x})$, that satisfies several basic mechanical constraints. Namely, the stress field is smooth, it satisfies equilibrium everywhere, and the surfaces are traction-free. These constraints are imposed explicitly on the residual stress distribution, $\Sigma^{h}(\boldsymbol{x})$. Smoothness is imposed via the choice of approximating functions; piece-wise, continuous, interpolation functions commonly used in FE methods are used here. The mechanical constraints of equilibrium and traction-free surfaces are imposed by penalizing any values in the stress distributions that would lead to deviations from local equilibrium and zero traction conditions. The mathematical representations of these constraints are provided on left side of Figure 10, where $\boldsymbol{t}(\boldsymbol{x})$ is the traction and $\boldsymbol{v}(\boldsymbol{x})$ is the surface normal. As indicated, the bi-scale optimization scheme also has the capability of imposing a symmetry condition. For the stress distribution, a symmetry condition implies that only the shear components of the traction are zero, whereas the free surface condition requires that all components of the traction are zero.

The values of the continuum distribution over the workpiece domain are chosen to match values deduced from experiment observations, namely the diffraction measurements. This is accomplished by minimizing a weighted residual, $R_{\Sigma}$, defined by the differences between the continuum stress distribution and a second spatial distribution, designated as $\Sigma^{d}(\boldsymbol{x})$, defined by interpolation of stress values deduced from the diffraction data. This residual is stated at the top of Figure 10 and is the mechanism through which the continuum scale mechanical constraints and the crystal scale lattice strain data are merged. The method employs a mesh-free method in representing $\Sigma^{d}(\boldsymbol{x})$, where the locations of the nodes of the mesh-free interpolation coincide with centroids of diffraction volumes.

As indicated on the right side of Figure 10 , the values of the stress at the nodes of the mesh-free interpolation are the diffraction volume averages, designated as $\langle\sigma\rangle$. There is one such average for every diffraction volume, which is evaluated as weighted integral of the crystal stresses, $\sigma(\boldsymbol{r})$, over a fundamental zone of the orientation domain, $\Omega_{\mathrm{fr}}$. The weighting factor in this integral is the orientation distribution function (ODF), $A(\boldsymbol{r})$. For the LSHR alloy examined in this investigation, a uniform ODF is assumed [31] based on measurements that indicated a weak texture. 
Weighted Residual on Stress Fields

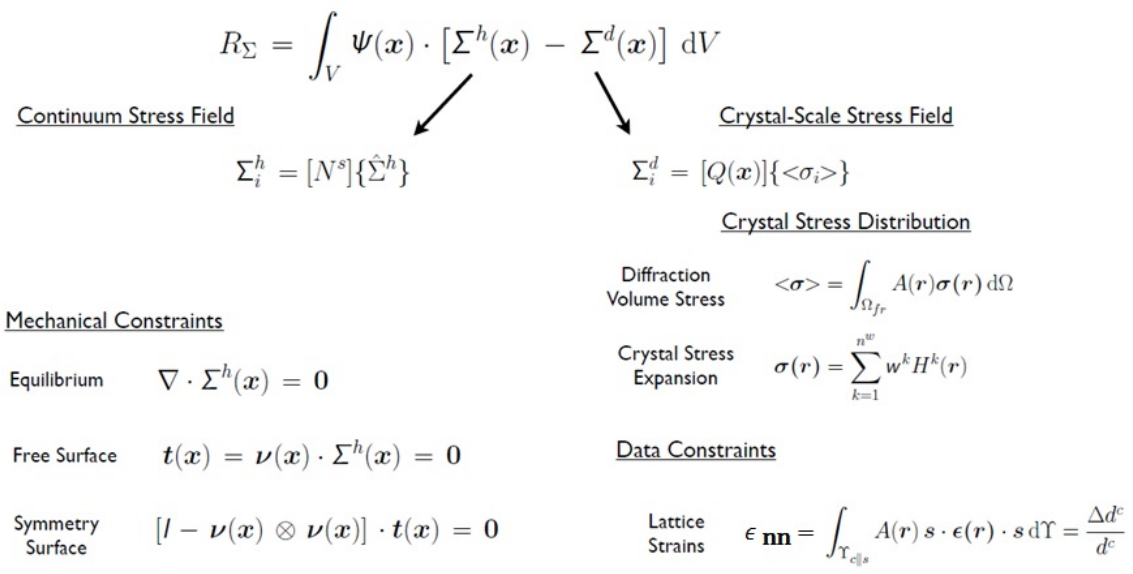

Solution Parameters

$\left\{\hat{\Sigma}^{h}\right\} \Rightarrow \begin{aligned} & \text { Continuum stress tensor parameters } \\ & \text { (one set for every node) }\end{aligned}$

$w^{k} \Rightarrow \begin{aligned} & \text { Crystal scale stress tensor parameters } \\ & \text { (one set for every diffraction volume) }\end{aligned}$

Figure 10: Schematic diagram showing key components of the finite element base data reduction methodology

$$
\begin{array}{ccc}
c_{11}(\mathrm{GPa}) & c_{12}(\mathrm{GPa}) & c_{44}(\mathrm{GPa}) \\
\hline \hline 245.25 & 155.16 & 125.00
\end{array}
$$

Table 2: The values of the single crystal elastic moduli used in the bi-scale optimization scheme [17] to compute the residual stress field.

The crystal-scale stress distributions are represented with a reduced-order parametrization based on a $k$-term harmonic expansion in which $H^{k}(\boldsymbol{r})$ are the harmonic modes and $w^{k}$ are the weights for the respective modes. The reduced-order representation provides a compact form for the crystal stress distribution with relatively few degrees of freedom.

The lattice strain data now enter the data reduction methodology. Measured lattice strains are related to the crystal elastic strains, $\boldsymbol{\epsilon}(\boldsymbol{r})$, via integration over the appropriate crystallographic fibers for application of Bragg's law. Here, $\Upsilon_{c \| s}$ refers to the fiber, or set of orientations, for which the crystal direction, $\boldsymbol{c}$, aligns with the sample direction, $\boldsymbol{s}$. Hooke's law is used to write the crystal elastic strains in terms of the crystal stresses, through which the single crystal elastic moduli enter. The moduli for the LSHR alloy are listed in Table 2.

The solution variables determined for the complete data reduction are the nodal stress values for the continuum mesh, $\left\{\hat{\Sigma}^{h}\right\}$, and the weights of the harmonic expansion for diffraction volume stress $w^{k}$. The diffraction volume average stress components can be recovered if desired, but are not explicitly needed. The continuum mesh of the hub and the boundary constraints used for the shrink fit application are shown in Figure 11

\section{Results and discussion}

The experimental SPFs reflect the state of stress in the disk. As described in 6 , the experimental SPFs drive the bi-scale optimization scheme employed to determine the 3D residual stress field in the disk. 


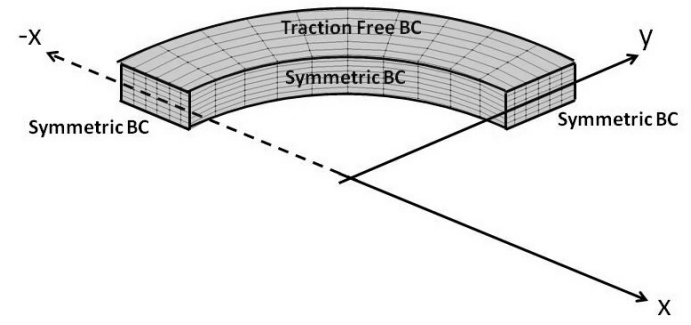

Figure 11: The FE mesh of the hub and the boundary conditions used for the bi-scale optimization scheme. The sample symmetry is exploited and appropriate boundary conditions are imposed.
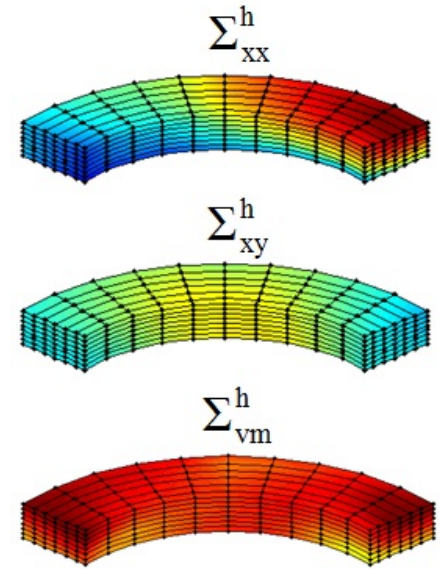
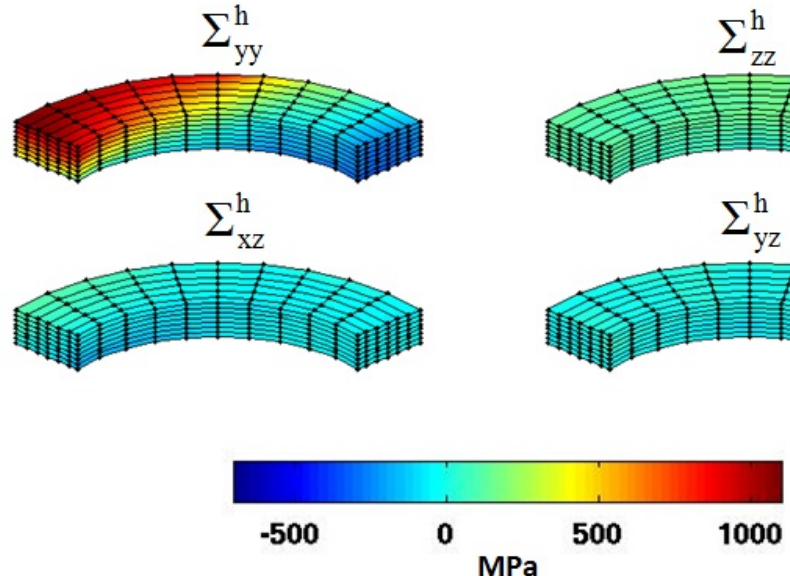
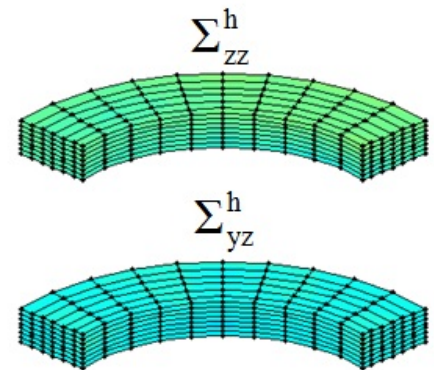

$\mathrm{MPa}$

Figure 12: The components of the 3D residual stress field and the von Mises effective stress of the disk obtained by combining the SPF data and the bi-scale optimization scheme are plotted over the FE mesh of the disk.

\subsection{D residual stress field in the disk obtained by the bi-scale optimization scheme}

Figure 12 shows the residual stress field in the disk determined from the SPF data set (Figure 8) and the bi-scale optimization scheme. Several trends stand out. First, $\Sigma_{x x}^{h}$ is compressive along the x-direction and tensile along the y-direction while $\Sigma_{y y}^{h}$ is tensile along the x-direction and compressive along the y-direction. This is consistent with the radial and hoop stresses that are expected to exist along the $\mathrm{x}$ - and $\mathrm{y}$ - directions on the sample. The xy-component of stress is the largest near $\alpha=45^{\circ}$ and approaches zero along the $\mathrm{x}$ - and $\mathrm{y}$-directions. The other components of stress $\left(\Sigma_{z z}^{h}, \Sigma_{x z}^{h}\right.$, and $\left.\Sigma_{y z}^{h}\right)$ are close to zero or the magnitudes are much smaller than those of $\Sigma_{x x}^{h}, \Sigma_{y y}^{h}$, and $\Sigma_{x y}^{h}$. These results are consistent with the trends shown in Figure 5 where the dominant stress components are $\Sigma_{x x}, \Sigma_{y y}$, and $\Sigma_{x y}$ while $\Sigma_{z z}, \Sigma_{x z}$, and $\Sigma_{y z}$ are close to zero.

Furthermore, the through-thickness stress gradient is well-resolved. The magnitudes of $\sum_{x x}^{h}, \Sigma_{y y}^{h}$, and $\Sigma_{x y}^{h}$ as well as the von Mises effective stress increase from the negative values of $z_{S}$ to the positive values of $z_{S}$ in general. This is consistent with the taper in the disk's inner diameter and sample assembly. The ability to capture not only the radial stress gradient but also the through-thickness stress gradient is the primary feature we set out to obtain and the results show the utility of this work.

Our 3D elastic FE approximation of the residual stress field (Figure 5) shows that the magnitudes of the stress components decrease monotonically with radial position. However, the magnitudes of $\Sigma_{x x}^{h}$ and $\Sigma_{y y}^{h}$ are not the largest at the inner-most radial position with the smallest disk radius (Figure 12). The bi-scale optimization method is driven by the experimental SPF data and the 3D residual stress field from the bi-scale optimization scheme reflects the SPF data. Figure 8 shows that the magnitudes of lattice strains do not decrease monotonically. For example, the SPFs for 


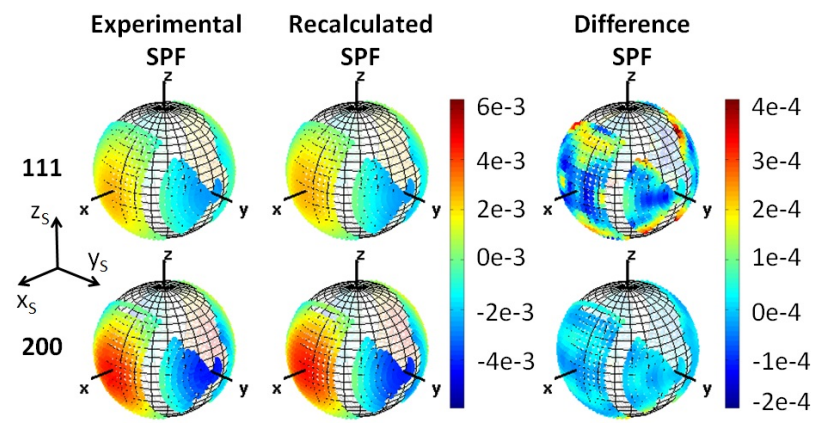

Figure 13: An example set of experimental SPFs and corresponding SPF recalculated via bi-scale optimization scheme. The experimental SPFs are from the diffraction volume located at $\left(\mathrm{r}_{\mathrm{S}}=7.80 \mathrm{~mm}, \alpha_{\mathrm{S}}=0^{\circ}, \mathrm{z}_{\mathrm{S}}^{\mathrm{SPF}}=0.255 \mathrm{~mm}\right)$. The difference between the experimental and recalculated SPFs is plotted as the difference SPFs. The experimental and reconstructed SPFs show a fair agreement.

$\left(\alpha_{\mathrm{S}}=0^{\circ}, \mathrm{z}_{\mathrm{S}}^{\mathrm{SPF}}=0.255 \mathrm{~mm}\right)$ show that the magnitudes of lattice strains do not decrease with $\mathrm{r}_{\mathrm{S}}$ monotonically. The magnitudes of lattice strain (especially close to the $\mathrm{x}$-direction) actually increase from $\mathrm{r}_{\mathrm{S}}=6.85 \mathrm{~mm}$ to $\mathrm{r}_{\mathrm{S}}=7.80 \mathrm{~mm}$ then decrease from $r_{S}=7.80 \mathrm{~mm}$ to $r_{S}=9.30 \mathrm{~mm}$. Reflecting this trend in the SPF, our residual stresses are not monotonically decreasing with respect to radius.

It is also worthwhile to note that the stress field depends on $\alpha_{\mathrm{S}}$, the angular position on the disk. If the stress field were independent of $\alpha$, the von Mises effective stress would be independent of $\alpha$. The von Mises effective stress in Figure 12 shows otherwise. This again is consistent with the strain pole figure measurements. While the location of the highs and lows in the strain pole figures depend on the Cartesian coordinate system that we chose, the magnitudes of the highs and lows in the strain pole figures taken at $\alpha=0^{\circ}$ and $\alpha=90^{\circ}$ at a particular radial position (Figures $8(\mathrm{a})$ and 8(b) for example) are similar but not identical.

\subsection{Recalculated SPFs and orientation dependent crystal stresses}

One way to check the accuracy of the 3D residual stress field obtained through the bi-scale optimization scheme is to reconstruct a set of SPFs from the bi-scale solution and compare them to the experimental SPF data. Figure 13 shows an example set of experimental SPF data and corresponding reconstructed SPFs. This figure shows the experimental $\mathrm{SPF}$ data for $\{111\},\{200\}$, and $\{220\}$ for diffraction volume located at $\left(\mathrm{r}_{\mathrm{S}}=7.80 \mathrm{~mm}, \alpha_{\mathrm{S}}=0^{\circ}, \mathrm{z}_{\mathrm{S}}^{\mathrm{SPF}}=0.255 \mathrm{~mm}\right)$ and corresponding reconstructed SPFs obtained from the bi-scale optimization. In this figure, the reconstructed SPFs and the experimental SPFs are in fair agreement. On average, the difference between the experimental SPF that drive the bi-scale optimization scheme and the reconstructed SPFs are smaller than $2 \times 10^{-4}$.

As a by-product of the bi-scale optimization scheme, the orientation dependent crystal stresses are also available. For a particular diffraction volume, the orientation dependent crystal stresses must satisfy the measured SPFs while satisfying the boundary conditions and equilibrium in the continuum length scale. An example of the orientation dependent crystal stresses are shown in Figure 14. In this figure, the components of the orientation dependent crystal stresses for the diffraction volume located at $\left(\mathrm{r}_{\mathrm{S}}=7.80 \mathrm{~mm}, \alpha_{\mathrm{S}}=0^{\circ}, \mathrm{z}_{\mathrm{S}}^{\mathrm{SPF}}=0.255 \mathrm{~mm}\right)$ are plotted over the cubic fundamental region of Rodrigues space. The crystal stresses are complicated; they are multi-axial and magnitudes vary significantly over orientation space. However, the complicated orientation dependent crystal stresses, when averaged appropriately over orientation space and used in conjunction with the bi-scale optimization scheme, enable us to determine the 3D residual stress field in a component. As other works [3], [47], [7] have indicated, making appropriate SPF measurements and interpreting them as crystallographic fiber measurements from crystals with anisotropic properties is important for determining the residual stress at the continuum scale.

The simulated residual stress field in the disk shown in Figure 5 is only an approximation of the actual 3D residual stress field in the disk. The isotropic elastic FE approximation does not reflect the exact boundary conditions of the actual sample and the exact amount of interference in the actual sample is unknown. Thus, the residual stress field shown in Figure 5 only serves as a qualitative guide to gauge whether the 3D residual stress field obtained via the bi- 


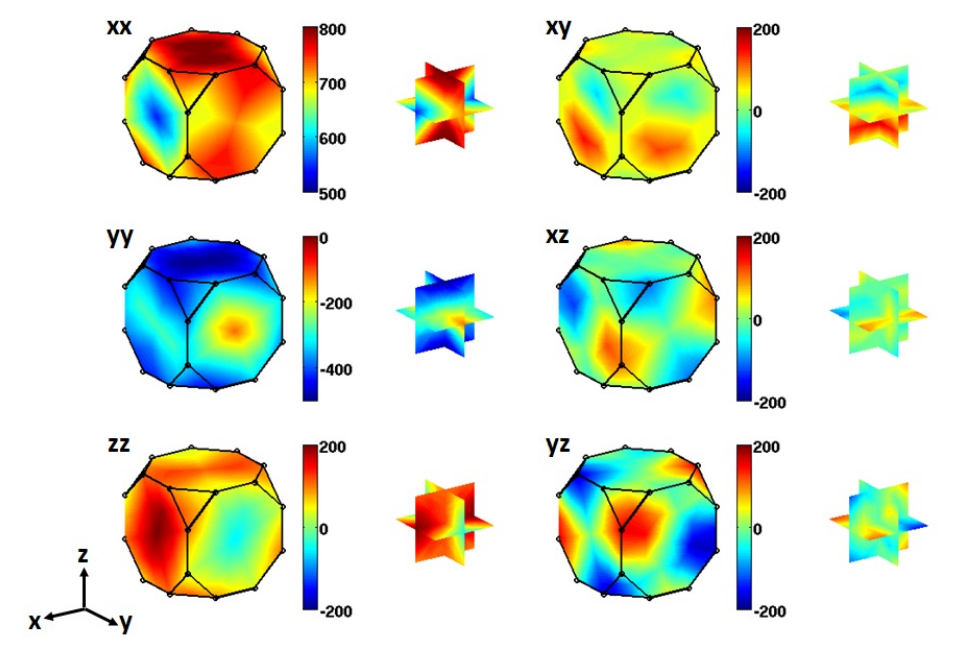

Figure 14: The orientation dependent crystal stresses for the diffraction volume located at $\left(\mathrm{r}_{\mathrm{S}}=7.80 \mathrm{~mm}, \alpha_{\mathrm{S}}=0^{\circ}\right.$, $\mathrm{z}_{\mathrm{S}}^{\mathrm{SPF}}=0.255 \mathrm{~mm}$ ) obtained from the bi-scale optimization scheme. The unit for the color bars is in MPa.

scale optimization scheme and the SPF data is reasonable. On the other hand, the SPF data reflect the state of residual stress in the disk. The bi-scale optimization scheme imposes appropriate boundary conditions and equilibrium while matching the SPF data using appropriate anisotropic single crystal moduli and crystallographic texture.

\subsection{Limitations of the methodology}

While the strain pole figures using the conical slits combined with the bi-scale optimization scheme presented so far are promising, there are several fronts where the current method can be improved to obtain a more reliable residual stress field.

It is worthwhile to note that the choice of the single crystal elastic moduli and the crystallographic orientation distribution will influence the 3D residual stress field obtained via the bi-scale optimization scheme and the SPF data. The anisotropic elastic constants relate the crystal scale strains to the crystal scale stresses in the bi-scale optimization scheme and appropriate constants need to be used. Using an appropriate crystallographic orientation distribution for each diffraction volume is also important as the bi-scale optimization scheme interprets the strain pole figures as the weighted fiber average of the orientation dependent crystal strains projected along scattering vectors where the weights are the crystallographic orientation distribution.

Because the bi-scale optimization scheme relies on the SPFs measured at individual diffraction volumes located throughout the sample to determine the 3D residual stress field in the sample, a sufficient number of grains needs to be present in a diffraction volume to obtain reliable orientation dependent stresses and strains [42]. In this work, the average grain size was $3 \mu \mathrm{m}$ and having a sufficient number of grains in a diffraction volume was not an issue. However, as we attempt to expand this methodology to real engineering applications with perhaps large grain sizes, having a sufficient number of grains may be a challenge. Furthermore, the residual stress in a diffraction volume cannot fluctuate significantly over the diffraction volume. These issues can be addressed by suitable sample translation and rotation schemes but are not implemented in this work.

A reliable set of reference lattice parameters is needed to obtain reliable SPFs. In this work, the magnitudes of the components of the residual stress field introduced to the disk are large and a small discrepancy in the reference lattice parameter does not change the SPFs significantly. However, in situations where the magnitudes of the components of the residual stress field are small, it is important to obtain a reliable set of reference lattice parameters [50].

The transmission geometry employed in this work implies that the thickness of the sample that can be investigated using the method presented here depends on the x-ray attenuation length of the sample. Since the conical slits impose the geometric condition, it is also possible to manipulate the wavelength of the monochromatic x-ray beam such that 
the higher order reflections utilize the slits that correspond to the lower order reflections so that thicker samples can be investigated.

The conical slits used in this work have a varying spatial resolution for different crystallographic planes because all slits have the same width. It is also possible to use different slit widths for different crystallographic planes such that the spatial resolutions for all crystallographic planes are identical. It is also emphasized that the conical slits used in this work are not collimators and allows us to measure strains as long as the diffraction peak width is smaller than approximately $\pm 0.5^{\circ}[27]$.

\section{Summary and conclusions}

The 3D residual stresses field within a component can be complicated. While it is a common practice to report a single component of stress in this multiaxial field, better estimates of the residual stress field will enable designers to accommodate for residual stress or even use the residual stress fields to an advantage. To obtain a full 3D residual stress field in a component, a non-destructive measurement method capable of probing inside a component is necessary. Furthermore, a suitable computation method that can properly utilize the measurements is needed.

In this work, a SPF measurement technique utilizing monochromatic synchrotron x-rays was modified to nondestructively isolate a particular diffraction volume located inside a component. The isolation of a particular diffraction volume inside a component was realized by the addition of the conical slits in the SPF measurement setup.

To demonstrate the new experimental method, an interference fit sample with a 3D stress gradient was manufactured by tapering the inner radius of the disk. Using the modified SPF measurement technique, strain pole figures from many diffraction volumes located throughout the disk were obtained. The data from the disk of the interference fit sample showed that the lattice strains are consistent with an anticipated stress field in the sample obtained using a 3D elastic FE approximation.

A residual stress determination scheme that links the continuum scale and the crystal length scale was introduced. In this bi-scale optimization scheme, the two length scales are linked by assuming that when crystal stresses at a material point are properly integrated over orientation space, it should sum to the stress in the continuum length scale. The SPF data provide information about the crystal stresses and they are linked through the crystallographic orientation distribution and the single crystal elastic moduli. The bi-scale optimization scheme also constrains the 3D stress field in the continuum length scale such that the physical boundary conditions are met.

The SPF data obtained from the interference fit sample were combined with the bi-scale optimization scheme and the $3 \mathrm{D}$ residual stress field in the disk was obtained. The residual stress field obtained through the combination of the bi-scale optimization scheme and the SPF data agrees favorably with the FE approximation of the stress expected to exist in the disk.

Using the new residual stress determination method introduced in this paper, the multiaxial residual stress field in a real component can be investigated. The effects of industrial practices that change the residual stress in a real component can be examined and the actual 3D field can be quantified.

\section{Acknowledgments}

This research was financially supported by the U.S. Air Force Office of Scientific Research Multi-Scale Structural Mechanics Program under contract number FA9550-09-1-0642. Use of the Advanced Photon Source was supported by the U.S. Department of Energy, Office of Science, Office of Basic Energy Sciences under contract number DEAC02-06CH11357. Professor Jim Williams of the Ohio State University is gratefully acknowledged for motivation and guidance of this work. The LSHR material used in this work was provided by Dr. T. J. Turner at the Air Force Research Laboratory (AFRL). The DPLAB Polycrystal Library (OdfPf) at Cornell University was used extensively for this work (http://anisotropy.mae.cornell.edu). 


\section{References}

[1] Albertini, G., Bruno, G., Dunn, B., Fiori, F., Reimers, W., Wright, J.: Comparative neutron and x-ray residual stress measurements on al-2219 welded plate. Materials Science and Engineering A 224, 157-165 (1997). DOI http://dx.doi.org/10.1016/S0921-5093(96)10546-3

[2] Allen, A., Hutchings, M., Windsor, C., Andreani, C.: Neutron diffraction methods for the study of residual stress fields. Advances in Physics 34(4), 445-473 (1985). DOI 10.1080/00018738500101791. URL http: //www.tandfonline.com/doi/abs/10.1080/00018738500101791

[3] Behnken, H.: Strain-function method for the direct evaluation of intergranular strains and stresses. Phys. Stat. Sol. 177, 401-418 (2000). DOI 10.1002/(SICI)1521-396X(200002)177:2〈401::AID-PSSA401〉3.0. CO;2-I. URL http://onlinelibrary.wiley.com/doi/10.1002/\%28SICI291521-396X\% 28200002\%29177:2\%3C401::AID-PSSA401\%3E3.0.CO;2-I/abstract

[4] Bell, T., Sun, Y.: Plasma surface engineering of low alloy steel. Materials Science and Engineering A 140, 419434 (1991). DOI http://dx.doi.org/10.1016/0921-5093(91)90458-Y. URL http://www. sciencedirect. com/science/article/pii/092150939190458Y

[5] Bernier, J., Park, J.S., Pilchak, A., Glavicic, M., Miller, M.: Measuring stress distributions in ti-6al-4v using synchrotron x-ray diffraction. Metall. Mater. Trans. A 39, 3120-3133 (2008). DOI 10.1007/s11661-008-9639-6

[6] Bernier, J.V., Miller, M.P.: A direct method for the determination of the mean orientation-dependent elastic strains and stresses in polycrystalline materials from strain pole figures. Journal of Applied Crystallography 39, 358-368 (2006). DOI 10.1107/S0021889806009873. URL/http://scripts.iucr.org/cgi-bin/ paper?cg5029

[7] Bernier, J.V., Miller, M.P., Park, J.S., Lienert, U.: Quantitative stress analysis of recrystallized ofhc cu subject to deformation in situ. Journal of Engineering Materials and Technology 130, 021,021 (2008). DOI 10.1115/1. 2870234

[8] Buchanan, D.J., John, R.: Relaxation of shot-peened residual stresses under creep loading. Scripta Materialia 59, 286-289 (2008). DOI http://dx.doi.org/10.1016/j.scriptamat.2008.03.021

[9] Bunge, H.: Texture Analysis in Materials Science - Mathematical Methods. Butterworths (1982)

[10] Chen, H., Yao, Y., Kysar, J.: Spatially resolved characterization of residual stress induced by micro scale laser shock peening. Journal of Manufacturing Science and Engineering 126(2), 226-236 (2004). DOI 10.1115/1. 1751189

[11] Cullity, B.D., Stock, S.R.: Elements of X-ray Diffraction. Prentice Hall (2001)

[12] Demir, E.: Determination of depth-resolved residual stress using lattice strain pole figure measurements. Computer Methods in Applied Mechanics and Engineering in review (2012)

[13] Fitzpatrick, M., Lodini, A. (eds.): Analysis of Residual Stress by Diffraction using Neutron and Synchrotron Radiation. Taylor and Francis (2003)

[14] Gabb, T.P., Gayda, J., Telesman, J., Kantzos, P.T.: Thermal and mechanical property characterization of the advanced disk alloy lshr. Tech. rep., NASA (2005). NASA/TM2005-213645

[15] Hauk, V.: Structural and Residual Stress Analysis by Nondestructive Methods: Evaluation - Application - Assessment, 1st edn. Elsevier Science (1997)

[16] Heinz, A., Neumann, P.: Representation of orientation and disorientation data for cubic, hexagonal, tetragonal and orthorhombic crystals. Acta Crystallographica Section A: Foundations of Crystallography A47, 780789 (1991). DOI 10.1107/S0108767391006864. URL/http://scripts.iucr.org/cgi-bin/paper? bx0517 
[17] Hosford, W.F.: Mechanical Behavior of Materials. Oxford University Press (1993)

[18] Hosford, W.F., Caddell, R.: Metal Forming. PTR Prentice Hall (1993)

[19] Hutchings, M.T., Withers, P.J., Holden, T.M., Lorentzen, T.: Introduction to the Characterization of Residual Stress by Neutron Diffraction. CRC Press (2005)

[20] Jacobus, K., Devor, R., Kapoor, S.: Machining-induced residual stress: Experimentation and modeling. Transactions of the ASME 122, 20-31 (2000). DOI 10.1115/1.538906

[21] Jun, T.S., Hofmann, F., Belnoue, J., Song, X., Hofmann, M., Korsunsky, A.M.: Triaxial residual strains in a railway rail measured by neutron diffraction. Journal of Strain Analysis 44(7), 563-568 (2009). DOI http: //dx.doi.org/10.1243/03093247JSA545

[22] Kobayashi, M., Matsuia, T., Murakami, Y.: Mechanism of creation of compressive residual stress by shot peening. International Journal of Fatigue 20, 351-357 (1998). DOI http://dx.doi.org/10. 1016/S0142-1123(98)00002-4. URL http://www.sciencedirect.com/science/article/pii/ S0142112398000024

[23] Korsunsky, A., Liu, J., Golshan, M., Dini, D., Zhang, S., Vorster, W.: Measurement of residual elastic strains in a titanium alloy using high energy synchrotron x-ray diffraction. Experimental Mechanics 46, 519-529 (2006). DOI 10.1007/s11340-006-8250-2

[24] Krawitz, A.D.: Neutron strain measurement. Materials Science and Technology 27(3), 589-603 (2011-0301T00:00:00). DOI doi:10.1179/1743284710Y.0000000029. URL http://www. ingentaconnect.com/ content/maney/mst/2011/00000027/00000003/art00001

[25] Kumar, A., Dawson, P.R.: Modeling crystallographic texture evolution with finite elements over neo-eulerian orientation spaces. Computational Methods in Applied Mechanics 153, 259-302 (1998). DOI http://dx.doi. org/10.1016/S0045-7825(97)00072-8. URL http://www.sciencedirect.com/science/article/ pii/S0045782597000728

[26] Lee, J., Aydiner, C., Almer, J., Bernier, J., Chapman, K., Chupas, P., Haeffner, D., Kump, K., Lee, P.L., Lienert, U., Miceli, A., Vera, G.: Synchrotron applications of an amorphous silicon flat-panel detector. Journal of Synchrotron Radiation 15, 477-88 (2008). DOI 10.1016/j.nima.2007.08.103

[27] Lienert, U., Martins, R., Grigull, S., Pinkerton, M., Poulsen, H., Kvick, A.: High spatial resolution strain measurements within bulk materials by slit-imaging. In: Mat. Res. Soc. Symp. Proc., vol. 590 (2000). DOI http://dx.doi.org/10.1557/PROC-590-241

[28] Lu, J.: Handbook of Measurement of Residual Stresses. The Fairmont Press (1996)

[29] Martins, R., Honkimaki, V.: Depth resolved strain and phase mapping of dissimilar friction stir welds using high energy synchrotron radiation. Textures and Microstructures 35, 145-152 (2003)

[30] Martins, R., Lienert, U., Margulies, L., Pyzalla, A.: Residual strain tensor determination within highly plastically deformed torsion samples using high energy synchrotron radiation. Journal of Nerutron Research 9 (2001)

[31] McNelis, K., Dawson, P., Miller, M.: A two-scale methodology for determining the residual stresses in polycrystalline solids using high energy x-ray diffraction data. Journal of Mechanics and Physics of Solids Volume 61, 428-449 (2013). DOI http://dx.doi.org/10.1016/j.jmps.2012.09.015. URL http: //www. sciencedirect. com/science/article/pii/s0022509612002086

[32] Menig, R., Pintschovius, L., Schulze, V., Voehringer, O.: Depth profiles of macro residual stresses in thin shot peened steel plates determined by x-ray and neutron diffraction. Scripta Materialia 45, 977-983 (2001). DOI http://dx.doi.org/10.1016/S1359-6462(01)01063-6. URL http://www.sciencedirect. $\mathrm{com} / \mathrm{science/article/pii/s1359646201010636}$ 
[33] Miller, M.P., Bernier, J.V., Park, J.S., Kazimirov, A.: Experimental measurement of lattice strain pole figures using synchrotron x rays. Review of Scientific Instruments 76, 113,903 (2005). DOI http://dx.doi.org/10.1063/ 1.2130668. URL http://rsi.aip.org/resource/1/rsinak/v76/i11/p113903_s1

[34] Nielsen, S., Wolf, A., Poulsen, H., Ohler, M., Lienert, U., Owen, R.: A conical slit for three-dimensional xrd mapping. Journal of Synchrotron Radiation 7, 103-109 (2000). DOI 10.1107/S0909049500000625. URL http://scripts.iucr.org/cgi-bin/paper?S0909049500000625

[35] Noyan, I.C., Cohen, J.B.: Residual stress : Measurement by Diffraction and Interpretation. Spinger-Verlag (1987)

[36] Pang, J.W.L., Holden, T., Wright, J., Mason, T.: The generation of intergranular strains in 309h stainless steel under uniaxial loading. Acta Materialia 48, 1131-1140 (2000). DOI http://dx.doi.org/10. 1016/S1359-6454(99)00382-1. URL http://www.sciencedirect.com/science/article/pii/ S1359645499003821

[37] Pollock, T.M., Tin, S.: Nickel-based superalloys for advanced turbine engines: Chemistry, microstructure, and properties. Journal of Propulsion and Power 22, 361-374 (2006). DOI 10.2514/1.18239

[38] Pratihar, S., Ganguly, S., James, J., Fitzpatrick, M., Edwards, L.: Non-destructive determination of the 3d residual stress in a aa7050 upper wing skin-stringer panel using neutron diffraction. Journal of Neutron Research 15, 293301 (2007). DOI 10.1080/10238160701374139. URL http://www.tandfonline.com/doi/abs/10. $1080 / 10238160701374139 \#$

[39] Prevy, P.S.: Metals Handbook, chap. X-ray diffraction residual stress techniques, pp. 380-392. American Society for Metals (1986)

[40] Sabol, G.P., Stickler, R.: Microstructure of nickel-based superalloys. Physica Status Solidi B: Basic Solid State Physics 35, 11-52 (1969). DOI 10.1002/pssb.19690350102. URL http: // onlinelibrary.wiley.com/ doi/10.1002/pssb.19690350102/abstract

[41] Said, A., Shastri, S.: Silicon saw-tooth refractive lens for high-energy x-rays made using a diamond saw. Journal of Synchrotron Radiation 17, 425-427 (2010). DOI 10.1107/S0909049510003584. URL http: / scripts. iucr.org/cgi-bin/paper?bf5028

[42] Schuren, J.C., Miller, M.: Quantifying the uncertainty of synchrotron-based lattice strain measurements. Journal of Strain Analysis 46, 663-681 (2011). DOI 10.1177/0309324711411553. URL http://sdj.sagepub. com/content/46/7/663.abstract

[43] Shigley, J., Mischke, C., Budynas, R., Nisbett, J.: Shigley's Mechanical Engineering Design, 8 edn. Tata McGraw-Hill (2008)

[44] Sims, C.: Superalloys: Genesis and character. In: C. Sims, N. Stoloff, W. Hagel (eds.) Superalloys II, chap. 1, pp. 3-26. John Wiley and Sons, Inc. (1987)

[45] Staron, P., Cihak, U., Stockinger, M., Clemens, H.: Characterization of residual stresses in in 718 turbine discs by neutron diffraction and finite element modeling. Journal of Neutron Research 15, 185-192 (2007). DOI 10.1080/ 10238160802399597. URL http://iospress.metapress.com/content/vr74251r1096u348/

[46] Wang, Y., Lin Peng, R., McGreevy, R.: A novel method for constructing the mean field of grain-orientationdependent residual stress. Philosophical Magazine 81(3), 153-163 (2001). DOI http://dx.doi.org/10.1080/ 09500830010017088

[47] Wang, Y., Lin Peng, R., Wang, X.L., McGreevy, R.: Grain-orientation-dependent residual stress and the effect of annealing in cold-rolled stainless steel. Acta Materialia 50, 1717-1734 (2002). DOI http://dx.doi.org/10. 1016/S1359-6454(02)00021-6. URL http://www.sciencedirect.com/science/article/pii/ S1359645402000216 
[48] Wanner, A., Dunand, D.C.: Synchrotron x-ray study of bulk lattice strains in externally loaded cu-mo composites. Metallurgical and Materials Transactions A: Physical Metallurgy and Materials Science 31(11), 2949-2962 (2000). DOI 10.1007/BF02830344

[49] Withers, P., Webster, P.: Neutron and synchrotron x-ray strain scanning. Strain 37, 19-33 (2001). DOI 10.1111/j.1475-1305.2001.tb01216.x. URL http://onlinelibrary.wiley.com/doi/10.1111/j. 1475-1305.2001.tb01216.x/abstract

[50] Withers, P.J., Preuss, M., Steuwer, A., Pang, J.W.L.: Methods for obtaining the strain-free lattice parameter when using diffraction to determine residual stress. Journal of Applied Crystallography 40, 891-904 (2007). DOI 10.1107/S0021889807030269. URL http://scripts.iucr.org/cgi-bin/paper?ks5141 